\title{
Metabolic dysregulation induces impaired lymphocyte memory formation during severe SARS-CoV-2 infection
}

Authors: Sanjeev Gurshaney ${ }^{1}$, Anamaria Morales Alvarez ${ }^{1}$, Kevin Ezhakunnel ${ }^{1}$, Andrew Manalo ${ }^{1}$,

${ }^{1}$ Cancer Division, Burnett School of Biomedical Science, College of Medicine, University of Central Florida, Orlando, FL 32827, USA

${ }^{2}$ Center for Cardiovascular Regeneration, Department of Cardiovascular Sciences, Houston Methodist Research Institute, Houston TX 77030

${ }^{3}$ AdventHealth Cancer Institute, AdventHealth Research Institute, Orlando, FL 32804

${ }^{4}$ Translational Research Institute, AdventHealth Research Institute, Orlando, FL32804, USA

\section{\# Corresponding Author:}

Hung Nguyen, PhD

6900 Lake Nona Blvd, Orlando, FL 32827, USA

Email: hung.nguyen@ucf.edu

Phone: (407)-266-7167

Fax: (407)-266-7017

Running title: Immunometabolic Landscape of COVID-19

Key words: COVID-19, SARS-CoV-2, COVID-19 biomarker, immunometabolism, lymphocytopenia, hypoxia, glycolysis, mitophagy, exhaustion, NK, NKT, epithelial cells

Conflict of interest: The authors declare no conflict of interest

\section{Abbreviations}

COVID-19, coronavirus disease 2019

SARS-CoV-2, severe acute respiratory system coronavirus 2

BALF, bronchoalveolar lavage fluid

PMBC, peripheral blood mononuclear cells

FAO, fatty acid oxidation

CRS, cytokine released syndrome

CTL, cytotoxic $T$ lymphocyte

SASP, senescence- associated secretory phenotype

EC, epithelial cells

Trm, T resident memory

ARDS, acute respiratory disease syndrome

p38 MAPK, p38 mitogen-activated protein kinase

LDHA, lactate dehydrogenase

PCA, principal component analysis

UMAP, universal manifold approximation and projection

CCA, canonical correlation analysis

ICU, intensive care unit 


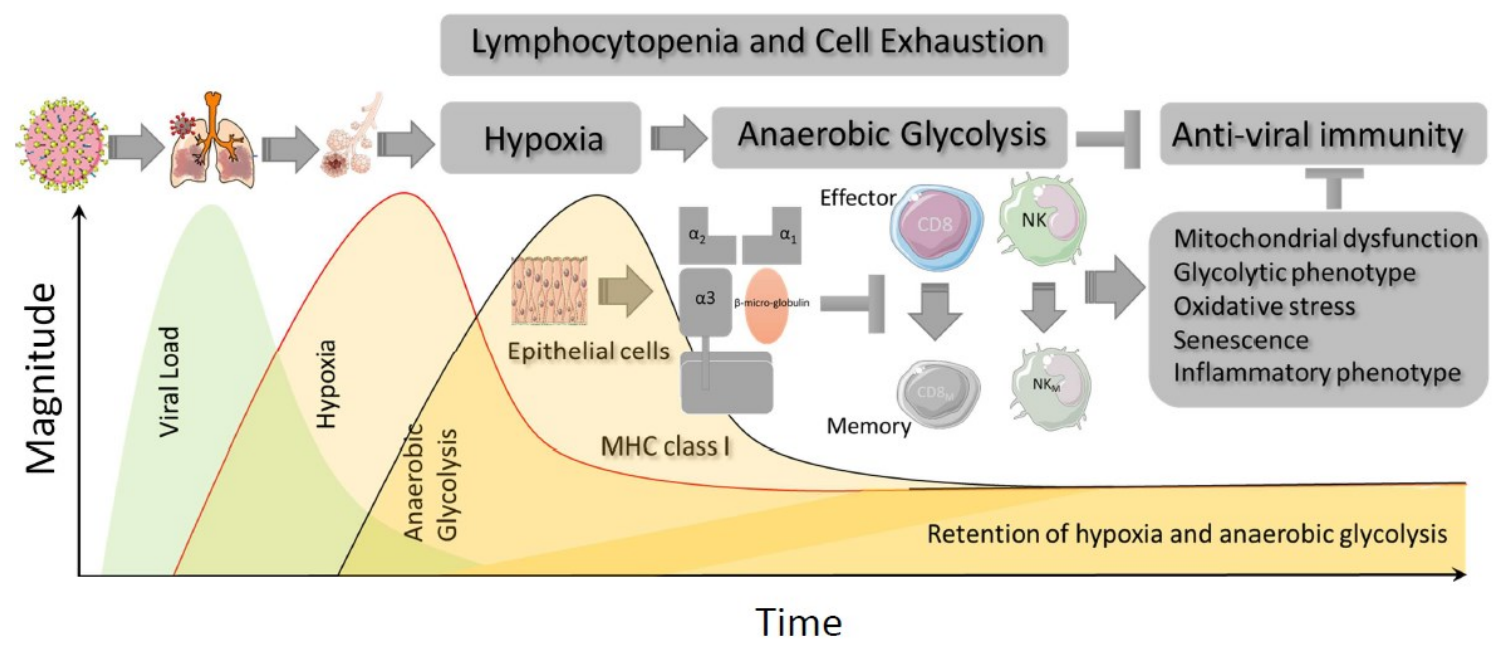

$47 \quad$ Highlights

- Hypoxia and anaerobic glycolysis drive CD8, NK, NKT dysfunction

- Hypoxia and anaerobic glycolysis impair memory differentiation in CD8 and NK cells cells 


\section{Abstract}

54 Cellular metabolic dysregulation is a consequence of COVID-19 infection that is a key determinant

55 of disease severity. To understand the mechanisms underlying these cellular changes, we 56 performed high-dimensional immune cell profiling of PBMCs from COVID-19-infected patients, in 57 combination with single cell transcriptomic analysis of COVID-19 BALFs. Hypoxia, a hallmark of 58 COVID-19 ARDS, was found to elicit a global metabolic reprogramming in effector lymphocytes. 59 In response to oxygen and nutrient-deprived microenvironments, these cells shift from aerobic 60 respiration to increase their dependence on anaerobic processes including glycolysis, mitophagy, 61 and glutaminolysis to fulfill their bioenergetic demands. We also demonstrate metabolic 62 dysregulation of ciliated lung epithelial cells is linked to significant increase of proinflammatory 63 cytokine secretion and upregulation of HLA class 1 machinery. Augmented HLA class-1 antigen 64 stimulation by epithelial cells leads to cellular exhaustion of metabolically dysregulated CD8 and 65 NK cells, impairing their memory cell differentiation. Unsupervised clustering techniques revealed 66 multiple distinct, differentially abundant CD8 and NK memory cell states that are marked by high 67 glycolytic flux, mitochondrial dysfunction, and cellular exhaustion, further highlighting the connection between disrupted metabolism and impaired memory cell function in COVID-19. Our findings provide novel insight on how SARS-CoV-2 infection affects host immunometabolism and anti-viral response during COVID-19. 


\section{Introduction}

77 According to the World Health Organization (WHO), over 3.6 million individuals across the world have perished due to the COVID-19 pandemic as of October $2021^{1}$. Although multiple SARSCoV-2 vaccines have been launched, herd immunity may not be reached in many countries until late 2021 or early $2022^{2}$. Additionally, mutant COVID-19 strains including the novel delta variant, which have the potential to partially evade immunity induced by currently available vaccines, as well as display significantly increased rates of infection are rapidly increasing in prevalence ${ }^{3}$. Therefore, novel therapeutics to combat SARS-CoV-2 are urgently needed.

Metabolic syndrome and its cluster of conditions pose risk factors for severe COVID-19

85 pathogenesis ${ }^{4,5}$. The growing body of evidence suggests that individuals with pre-existing metabolic comorbidities are at far higher risk of suffering severe complications from COVID-19 6,7 . However, understanding about the metabolism of immune cells in the microenvironment of injured organs such as the lung during SARS-CoV-2 infection is limited. Most studies have been performed on patients' peripheral blood mononuclear cells (PBMCs) ${ }^{4,5,8}$. Because the metabolic characteristics of the target organ and circulation are different ${ }^{9}$, knowledge of the immunometabolic landscape in injured organ by SARS-CoV-2 is essential for generating safe and effective treatments for COVID-19. Additionally, metabolic biomarkers, used stand-alone or in combination, that specifically predict the prognosis of COVID-19 may provide the essential knowledge for clinicians to triage care accordingly if needed.

The lung is the primary target organ of SARS-CoV-2, as the spike protein directly binds to ACE2 receptors expressed on the surface of lung epithelial cells $(E C s)^{10}$. As a result, acute respiratory

97 distress syndrome (ARDS) often occurs in severe COVID-19, resulting in decreased blood oxygen 98 saturation level (hypoxia), as well as increased serum lactate dehydrogenase (LDHA) levels ${ }^{11-13}$. Both downstream hypoxia signaling and hyperlactatemia have been associated with 
101 completely understood whether/how hypoxia in COVID-19 ARDS patient affects the metabolic 102 phenotype of immune cells. Thus, it is essential to identify metabolic dysregulation responsible 103 for immunological impairment during severe COVID-19.

104 In the current study, a publicly available single cell sequencing dataset from the bronchoalveolar 105 lavage fluid (BALF) of COVID-19 patients was used to generate a transcriptional landscape of 106 metabolic activity at a single-cell resolution in COVID-19 lung microenvironment. We also 107 performed high- dimensional flow cytometry of hospitalized COVID-19 patient PBMCs to validate 108 the results at the protein level. We found that metabolic disorder by hypoxia and anaerobic 109 glycolysis impairs memory differentiation in CD8 and NK cells during SARS-CoV-2 infection. 110 Multiple distinct, highly resolved CD8 and NK cell subsets with metabolic and mitochondrial 111 dysfunction were found to effectively indicate COVID-19 severity. The findings in current study 112 provide a single-cell metabolic landscape of COVID-19, highlighting the metabolic plasticity and 113 heterogeneity of immune cells, which linked to host immunity against SARS-CoV-2 thereby having 114 translational application for COVID-19 severity assessment, treatment, and therapy. 
123

124

125

126

127

128

129

130

131

132

133

134

135

136

137

138

139

140

141

142

\section{Results}

High-dimensional flow cytometry and BALF transcriptomic analysis reveal cellular dysfunction and impaired memory formation in CD8 T and NK cells in patients with COVID19.

Immunophenotyping of PBMCs from hospitalized COVID-19 patients was performed to probe the immunological response and metabolic perturbations that arise from SARS-CoV-2 infection (Fig. 1A). PBMCs were freshly isolated from 20 COVID-19 patients and 8 control subjects and analyzed without stimulation or cryogenic preservation (Fig. 1A). Given their critical roles in the primary immune response against SARS-CoV-2, we initially start with CD8 ${ }^{+} \mathrm{T}$ and $\mathrm{NK}$ cells ${ }^{16,17}$. Principal component analysis (PCA) revealed distinct clustering of COVID-19 and healthy control samples, thus revealing that PBMCs from COVID-19 patients exhibited an abnormal immunophenotype (Fig. 1B). FlowSOM unsupervised clustering and universal manifold approximation projection (UMAP) dimensionality reduction were next performed to detect unsupervised clusters. UMAP embedding confirmed distinguished immunophenotype of PBMC from COVID-19 patient and healthy control (Fig. 1C). Unsupervised clusters were annotated based upon canonical marker expression (Supplementary Fig. S3A-B) to define distinct and highly resolved cell populations (Fig. 1D, E). Cytotoxic T lymphocytes (CTLs) were identified by coexpression of CD8 and GZMB; CD8 central memory by CD8, CD62L, and CCR7; CD8 transitional memory by CD8, CD62L; CD8 effector memory by CD8, CCR7, GZMB; NK by CD56; NKT by CD56, CD8; memory NK (CD62L ${ }^{+}$NK) by CD56, CD62L (Supplementary Table 3).

Nonparametric differential abundance testing showed significantly decreased levels of CD8 transitional memory and memory NK cells in PBMCs from COVID-19 patients (Fig. 1F) Additionally, the percentages of CTLs and NKT were remarkably reduced in COVID-19 PBMCs. These findings suggest that severe COVID-19 infection may be associated with impaired lymphocyte memory formation and accompanying immunological dysfunction. 
We also examined if these changes were also identified in cells harvested from the lungs of COVID-19 patients, the primary target for virus infection, using publicly available single cell RNAsequencing data from BALF samples ${ }^{18}$. Louvain optimization and UMAP dimensionality reduction were applied to generate unsupervised clusters (Fig. 1G) which were then annotated by expression of canonical markers (Supplementary Fig. 1A, B) to define highly resolved populations

(Fig. 1H). Canonical markers used to annotate the unsupervised clusters and define cell subsets were as follows: $C D 8^{+} \mathrm{T}$ cells $(C D 3 D$ and $C D 8 A) ; C D 4^{+} \mathrm{T}$ cells $(C D 3 D$ and $C D 4) ;$ proliferating $\mathrm{T}$ cells (CD3D and MKI67); pseudostratified epithelial cells (EPCAM, CFAP126, and DNAAF1); nonciliated epithelial cells (EPCAM); plasma cells (IGKC and MS4A1(-)); B cells (MS4A1); neutrophils (HCAR3); NK cells (KLRC1); MAST cells (LTC4S); pDCs (CLEC4C); myeloid dendritic cells (mDCs) (CD1C and CLEC9A); macrophages (CD68 and FABP4); peripheral monocytes (CD68, FCN1, and CD14) (Supplementary Fig. 1A-B).

After initial cell type identification, T cells were subsetted and unsupervised clustering was performed again to achieve higher resolution into distinct $T$ cell lineages (Fig. $\mathbf{1 I}, \mathbf{J}$ and Supplementary Fig. 2A). Abundance of CD8 memory and NKT cells was found reduced and positively correlated to disease severity (Fig. 1K). Taken together, these results suggest that memory differentiation of CD8 ${ }^{+} \mathrm{T}$ and NK cells is impaired in both the lungs and in circulation of COVID-19 patients.

\section{Hypoxia/anaerobic glycolysis axis causes cellular dysfunction of CTLs in COVID-19.}

167 Expression of cellular markers for metabolism and exhaustion was evaluated in CD8 T cells from COVID $-19^{+}$hospitalized patients to gain insight about the mechanistic underpinnings of cellular dysfunction. Increased HIF-1 $\alpha$ expression in CD8 T cells is indicative of hypoxia, a condition that accompanies pulmonary damage in COVID-19 patients (Supplementary Fig. 4A). Increased

171 glycolytic dependence of CD8 T cells from COVID-19 patients was suggested by the upregulation

172 of 2-(2-(N-(7-Nitrobenz-2-oxa-1,3-diazol-4-yl)Amino)-2-deoxyglucose) (2-NBDG) uptake 
173 (Supplementary Fig. 4B). The glucose transporter 1 (GLUT1) has been well known to govern

174 glycolytic flux ${ }^{19}$ and participates in the generation of cytotoxic CD8 lymphocytes (CTLs) ${ }^{20}$. The

175 frequency and expression of GLUT1 in CTLs present in PBMCs from COVID-19 patients were

176 significantly increased (Supplementary Fig. 4C, D), suggesting that these cells are phenotypically

177 glycolytic. Under hypoxic conditions, a metabolic phenotype primarily dependent on anaerobic

178 glycolysis often leads to oxidative stress and mitochondrial dysfunction ${ }^{21}$. Indeed, the frequency

179 of $\mathrm{ROS}^{+} \mathrm{CTL}$ was elevated in PBMCs from COVID-19 patients (Supplementary Fig. 4E).

180 Furthermore, GLUT1 ${ }^{+}$CTL of COVID-19 patients expressed higher levels of VDAC-1

181 (Supplementary Fig. 4F). which is an indicator of reactive oxidative species (ROS) generation and

182 mitochondrial death ${ }^{22}$. This metabolic dysregulation in COVID-19 CTLs is likely linked to cellular

183 exhaustion, as indicated by the upregulation of LAG-3 in this cell subset. (Supplementary Fig.

184 4G). To further establish the interplay between glycolysis, mitochondrial dysfunction, and cellular

exhaustion in CTLs, we performed unsupervised clustering specifically on metabolic and functional markers to glean insight into the metabolic state of CTLs (Supplementary Fig. 3C). We observed and defined a unique cluster displaying high expression of GLUT1, LAG-3, ROS, and VDAC1, indicative of elevated glycolysis, exhaustion, and mitochondrial stress, as GLUT1 ${ }^{+}$ mitochondrially exhausted CTLs (Supplementary Fig. 4H). This cell population was enriched in severe COVID-19 PBMCs (Supplementary Fig. 4I). These results indicate that mitochondrial and metabolic dysfunction that is closely tied to CTL exhaustion in COVID-19 patients.

192 The BALF transcriptomic dataset also revealed increased expression of genes encoding 193 anaerobic glycolysis in CTLs from moderate (M)- and severe (S)- COVID-19 patients 194 (Supplementary Fig. 5A,B). Expression of transcripts coding for glycolytic enzymes including 195 GAPDH, GALM, and ALDOA was significantly increased in COVID-19 CTLs (Supplementary Fig. 5A,B). Key metabolic pathways including hypoxia, anaerobic glycolysis, mitophagy, autophagy, cell exhaustion, and senescence were upregulated, while pathways relying on mitochondrial 
metabolism including fatty acid oxidation (FAO), cholesterol metabolism, and OXPHOS were attenuated in CTLs from COVID-19 patients (Supplementary Fig. 5A,C). The increased glycolytic state in S-COVID-19 CTLs was revealed by GSEA analysis coupled with the overexpression of key glycolytic regulator genes (GAPDH, GALM, ALDOA) (Supplementary Fig. 5A,B). Hierarchical clustering of differentially expressed glycolytic genes suggested a tight association between HIF$1 \alpha$ expression and anaerobic glycolysis (Supplementary Fig. 5B), indicating that oxygen-deprived condition in the BALF environment links to glycolytic metabolism. During normal CTL differentiation, cells preferentially shift from OXPHOS towards mTOR/ATK-mediated aerobic respiration to sustain increased bioenergetics demand and augmented mitochondrial biosynthesis $^{23}$. GSEA analysis showed that CTLs are less dependent on mitochondrial TCA metabolism when impacted by SARS-CoV-2 infection (Supplementary Fig. 5C). Reduction of $\mathrm{NAD}^{+}$to $\mathrm{NADH}$ conversion is required to preserve cellular redox homeostasis and sustain glycolytic flux. We observed decreased expression of transcripts encoding NADH oxidoreductases (NDUFB8, NDUFC2, and NDUFA11) in CTLs from COVID-19 patients

212 (Supplementary Fig. 5A,C). There was also downregulation of lipid metabolism- associated genes 213 (FABP4, APOC1, APOE, MARCO) in COVID-19 CTLs (Supplementary Fig. 5A,C). Increased 214 oxidative stress in COVID-19 CTLs was evident by overexpression of NFE2L2 and PRDX2 215 (Supplementary Fig. 5D). Decreased NADH oxidation and a concomitant increased NAD ${ }^{+}$level is associated with impaired cytokine secretion, cell proliferation, and exhaustion ${ }^{24,25}$. Indeed, the

21819 CTLs (Supplementary Fig. 5A,B). These data reveal that hypoxia- induced CD38 expression 219 is associated with metabolic reprograming and cellular exhaustion of CTLs in the lung of COVID22019 patients. This conclusion is supported by higher levels of exhaustion marker LAG3 in CTLs 221 from S- COVID-19 patients (Supplementary Fig. 5A,B). Taken together, these results suggest 222 that hypoxia arising from COVID-19-pulmonary dysfunction triggers impaired FA metabolism and 223 oxidative stress, which induces mitochondrial dysfunction and cellular exhaustion. 
225 Memory cell differentiation of CD8 T cells is a critical component of the immunological response 226 against viral re-infection ${ }^{26}$. To better understand the kinetics and dynamics of CD8 memory cell 227 differentiation during SARS-CoV-2 infection, we performed trajectory inference and 228 pseudotemporal modeling analysis on BALF CD8 T cells (Fig. 2A). Differential analysis revealed decreased pseudotime values for CD8 memory in S compared to M- COVID-19 and healthy control (Figs. 2A, B). The frequencies of proliferating CD8 and GNLY+ effector CD8 T cells were significantly increased in severe COVID-19 compared to healthy controls (Fig. 2C). Moreover,

232 CD8 cells were found highly proliferating in M- than S- COVID-19 patients (Fig. 2C). In contrast, a lower abundance of $\mathrm{GNLY}^{+}$effector CD8 cells was found in S-COVID-19. These findings suggest that CD8 T cells are stalled along the memory differentiation trajectory and are unable to reach the terminal state during S- COVID-19 infection.

During viral infection, circulating effector memory cells migrate to the infected tissue and differentiate into tissue-resident memory $\left(T_{\mathrm{RM}}\right)$ cells to provide the first response defense against reencounter of the pathogen ${ }^{27}$. Consistently, CD8 effector memory (CD8 ${ }_{\mathrm{EM}}$ ) cells expressed higher tissue residence phenotype (i.e., increased expression of ITGA1 and ZNF683) in M- as compared to S- COVID-19 patients (Fig. 2D). This finding may indicate that impaired CD8 memory differentiation is driving factor resulting in impaired viral clearance during severe COVID19. In contrast, trajectory inference and pseudotemporal ordering revealed no significant difference between the differentiations of CD4 cells amongst conditions (Supplementary Figs. 7A, B), suggesting that CD4 T cells retain proper differentiation and memory formation despite the metabolic stress during SARS-CoV-2 infection.

247 CD8 $\mathrm{T}$ cells were identified as memory cells based on expression of HOPX and SELL 248 (Supplementary Fig. 3A). GSEA analysis revealed that CD8 ${ }_{M}$ cells from S- or M- COVID-19 
patients were highly dependent on glycolysis for their energetic demands (Figs. 3A, B). Close clustering and shared upregulation of glycolytic enzyme coding genes GALM, GAPDH, GPI, and ALDOA with HIF1A, and transcripts regulating exhaustion (TIGIT and LAG3) (Fig. 3B), indicated that hypoxia and anaerobic glycolysis are associated with impaired CD8 ${ }_{M}$ function in COVID-19 BALF. FAO oxidation and OXPHOS promote the development of $\mathrm{CD} 8_{\mathrm{M}}$ cells after antigen exposure $^{28}$. Indeed, genes encoding regulators of lipid uptake (APOE, and APOC1) and FAO (OLR, MARCO, FABP4, PPRY) were downregulated in CD8 ${ }_{\mathrm{M}}$ cells of S-COVID-19 patients (Figs.

3A, C). Decreased expression of OXPHOS coding genes was found in S-COVID-19 CD8 ${ }_{\mathrm{M}}$ cells Pearson correlation analysis revealed a negative correlation $(R=-0.73, p=0.011)$ between module scores for glycolysis and FAO (Fig. 3D), which further showed a potential association between prolonged anaerobic glycolysis and reduced mitochondrial fitness. Moreover, a strong positive correlation between module scores for glycolysis and exhaustion $(R=0.85, p=0.00026)$

(Fig. 3D) validates that excessive glycolytic dependence is likely tightly linked to $C D 8_{M}$ exhaustion. Genes involved in cellular senescence and mitophagy were also upregulated in SCOVID-19 CD8 ${ }_{M}$ cells (Fig. 3A), implying that CD8 ${ }_{M}$ cells metabolically switch to these pathways to satisfy bioenergetics demands in response to impaired mitochondrial metabolism. Likewise, 267 glutaminolysis is also used as an alternative bioenergetic pathway, evident by upregulation of 268 glutamate oxidation regulating genes GLUD1 and DGLUCY in CD8 ${ }_{M}$ cells during COVID-19, likely 269 in response to reduced lipid uptake and FAO (Fig. 3A).

270 PCA analysis performed on 30 differentially expressed metabolic genes (Table S1) showed 271 distinct clustering of $C D 8_{M}$ cells across different groups, further highlighting the potential of these 272 pathways to be used as predictive markers for disease severity (Fig. 3E). In this line, Pearson 273 correlation analysis showed a strong positive correlation between expression of genes regulating 
274 glycolysis, mitophagy, senescence, and glutaminolysis (Fig. 3F). These genes were inversely 275 correlated with transcripts regulating FAO and NADH oxidation (Fig. 3F). Finally, increased CD38 276 expression in S- COVID-19 CD8 ${ }_{M}$ cells was closely clustered with exhaustion-coding genes (LAG2773 and TIGIT) (Fig. 3B) suggesting CD38 expression is associated with metabolic reprograming, 278 memory impairment and cellular exhaustion of $C D 8_{M}$ in the lung of COVID-19 patients.

279 Because CD8 memory cell differentiation is highly dependent on their metabolism ${ }^{29}$, we assessed 280 the metabolic profile of $\mathrm{CD} 8_{\mathrm{M}}$ cells from patient-derived PBMCs. Increased abundance of 281 GLUT1 $^{+}{ }^{+}$D8 $8_{M}$ cells in COVID-19 PBMCs validated the glycolytic phenotype of CD8 ${ }_{M}$ cells in 282 COVID-19 (Fig. 4A). GLUT1 ${ }^{+}$CD8 ${ }_{M}$ cells exhibited increased ROS expression (Fig. 4B), which might reflect elevated oxidative stress in COVID-19 PBMCs. Upregulation of LAG-3 in COVID-19 GLUT $1^{+} \mathrm{CD} 8_{\mathrm{M}}$ cells was indicative of cellular exhaustion in addition to memory cell impairment in COVID-19 CD8 ${ }_{M}$ cells (Fig. 4C). Despite no apparent change in $\mathrm{ROS}^{+} \mathrm{CD}_{\mathrm{M}}$ cell abundance between diseases states (Fig. 4D), these cells indeed exhibited augmented HIF-1a and VDAC expression, suggesting hypoxia-induced mitochondrial dysfunction in this cell subset during COVID-19 infection (Fig. 4E,F). Downstream unsupervised clustering on metabolic markers revealed increased abundance of GLUT1 ${ }^{+}$mitochondrially exhausted CD8 ${ }_{M}$ in COVID-19 PBMCs

(Fig. 4G). Together, these data demonstrate that the hypoxia/anaerobic glycolysis axis mediates 291 CD8 ${ }_{M}$ cellular dysfunction and exhaustion in COVID-19.

\section{Aberrant metabolism causes NKT dysfunction in COVID-19 patients}

NKT cells which express CD56 and CD8 are intermediate between the CD8 and NK cell lineages. NKT cells play critical roles in preventing pneumonia during chronic pulmonary disease ${ }^{30}$. There were reduced levels of circulating and BALF NKT cells in severe COVID-19 patients (Figs. 1F).

296 We examined the potential link between aberrant cellular metabolism and NKT lymphocytopenia 297 in COVID-19. The frequency of glycolysis dependent GLUT1 ${ }^{+}$NKT cells are more abundant in 298 COVID-19 patients (Supplementary Fig. 8A). There was also a striking increase in the frequency 
of $\mathrm{ROS}^{+} \mathrm{NKT}$ cells in COVID-19 PBMCs (Supplementary Fig. 8B). Interestingly, these cells displayed elevated HIF-1 $\alpha$ expression (Supplementary Fig. 8B), which further implied that hypoxia also triggers oxidative stress in NKT cells during COVID-19 infection. To further establish the relationship between this altered metabolism and cellular function, a secondary unsupervised clustering was performed. We identified a population of GLUT1 ${ }^{+}$mitochondrially exhausted NKT cells, representative of combined augmented glycolytic phenotype, impaired mitochondrial function, and cellular exhaustion, that were significantly increased in COVID-19 patients (Supplementary Fig. 8C). Higher HIF-1 $\alpha$ expression in this cell subset further validated that hypoxia-induced glycolysis is a key mechanism underlying NKT mitochondrial dysfunction (Supplementary Fig. 8C). In summary, these results suggest that NKT cells in circulation acquire significant metabolic-induced cell dysfunction because of prolonged exposure to hypoxic conditions in COVID-19.

311 To further probe the perturbed metabolism in NKT cells from COVID-19 patients, we characterized gene expression profiles of BALF NKT. Co-expression of CD8A and KLRD1 was used to define NKT cell lineage (Supplementary Fig. 3A). A transcriptional program associated with hypoxia- induced metabolic reprogramming was seen in NKT cells from S COVID-19 patients (Supplementary Fig. 8D). Normally, activated NKT cells use pyruvate dehydrogenase (PDHA1/2) to supply acetyl-CoA to the TCA cycle ${ }^{31}$. However, the oxygen- deprived environment in the lung

317 of COVID-19 patients was found to promote downregulation of genes encoding NADH oxidation 318 (NDUFB8, NDUFA11, NDUFA13), as well as FAO and lipid uptake (Supplementary Fig. 8D,E). 319 Consequently, NKT cells are dependent on anaerobic glycolytic metabolism to fulfil their 320 bioenergetic demands (Supplementary Fig. 8D,F). However, expression of genes- regulating 321 OXPHOS and TCA cycle were upregulated, suggesting that mitochondrial fitness was likely not 322 affected in COVID-19 NKT cells (Supplementary Fig. 8D,E). This observation further revealed 323 that OXPHOS was insufficient to support NKT effector function under COVID-19 hypoxic 
324

325

326

327

328

329

330

331

332

333

334

335

336

337

338

339

340

341

342

343

344

345

346

347

conditions. Alternatively, increased amounts of GADD45B and SLC25A5 transcripts (Supplementary Fig. 8E) indicated that NKT cells undergo metabolic adaptation via enhancement of mitophagic activity to produce basal catabolic intermediates required for effector cytokine secretion.

\section{Dysregulated metabolism promotes memory CD62L ${ }^{+}$NK cell exhaustion in COVID-19} patients

CD62 $\mathrm{L}^{+} \mathrm{NK}$ cells display memory-like attributes, evident by their rapid response to secondary viral infection and subsequent transformation to effector subtypes ${ }^{32}$. Decreased percentages of CD62L $^{+}$NK cells in COVID-19 PBMCs (Fig. 5A) prompted us to investigate the impact of cellular metabolism on $\mathrm{CD} 62 \mathrm{~L}^{+} \mathrm{NK}$ survival and function. The frequency of $\mathrm{GLUT1} 1^{+} \mathrm{CD} 62 \mathrm{~L}^{+} \mathrm{NK}$ was increased in COVID-19 PBMCs (Fig. 5B). Importantly, GLUT1 ${ }^{+}$CD62L $^{+}$NK frequency was positively correlated with serum glucose levels $(R=0.76, p=0.0042)$ (Fig. 5C), which unveils a pivotal relationship between blood glucose levels and memory cell glucose uptake. GLUT1 ${ }^{+}$ $\mathrm{CD} 2 \mathrm{~L}^{+} \mathrm{NK}$ also expresses significantly higher levels of HIF-1 $\alpha$ (Fig. 5D) and ROS (Fig. 5E), suggesting that hypoxia- mediating anaerobic glycolysis induces oxidative stress in $\mathrm{CD}^{2} \mathrm{~L}^{+} \mathrm{NK}$ cells during COVID-19 infection. This interpretation was supported by upregulation of HIF-1 $\alpha$ in ROS $^{+} \mathrm{CD} 2 \mathrm{~L}^{+} \mathrm{NK}$ cells (Fig. 5F). Given that elevated GLUT1 expression is associated with lymphocyte exhaustion and mitochondrial dysfunction, these results may suggest that hyperglycemic COVID-19 infected patients will likely exhibit exacerbated CD62L ${ }^{+} \mathrm{NK}$ cell dysfunction because of hypoxia-driven glycolytic metabolic reprogramming.

\section{Metabolic dysregulation impairs immune surveillance and increases proinflammatory} response in lung epithelial cells during SARS-CoV-2 infection.

Epithelial cells (ECs) secrete cytokines and help mediate antigen presentation in order to modulate immune cells function during viral infection ${ }^{33}$. Differential expression analysis revealed 
overexpression of key immune signaling pathways in COVID-19 ECs (Figs. 6A). Network analysis demonstrates a connection of COVID-19 infection with the downregulation of the transcriptional factors, ZKSCAN1 and CSNK2B, and upregulation of KLF6, NEAT1, and JUND (Figs. 6B). Induction of a pro-inflammatory cascade including type 1 IFN, toll-like receptor, NF-kB, and chemokine signaling and PI3K/AKT pathway was observed in COVID-19 ECs (Figs. 6C,G). Glucose metabolism mediates type I IFN secretion through enhancing transcriptional expression and epigenetic acetylation ${ }^{34}$. Indeed, we found a positive correlation between module scores for glycolysis and type 1 IFN signaling, as well as for glycolysis and NF-kB signaling (Figs. 6D, E).

356 Chronic presentation of viral antigens to CD8 T cells by ECs may cause cellular dysfunction ${ }^{35}$. 357 We observed that genes encoding HLA class 1 (HLA-E, PSMA-6, TAP1, IFI30) were enriched in 358 COVID-19 ECs (Fig. 6A). GSEA analysis further confirmed the upregulation of HLA class 1 antigen presentation in bulk ECs. In contrast, downregulation of genes encoding HLA class 2 (HLA-DRA, HLA-DPA1, HLA-DMA, DYNLL1) was found in COVID-19 ECs (Fig. 6A) which was further confirmed by GSEA analysis (Fig. 6F). Glycolysis was reported to repress functional response of antigen presenting cells during infection ${ }^{36}$. We indeed observed a negative correlation of glycolysis and genes encoding HLA class 2 machinery (Fig. 6H). These results revealed potential links between dysregulated EC metabolism with cytokine release syndrome and adaptive immune dysfunction in COVID-19.

BALF ECs were next identified and subsetted for downstream analysis(Fig. 7A). Differential expression analysis revealed key differences in the expression of transcripts governing key metabolic pathways (Fig. 7B). Additionally, UMAP performed solely on differentially expressed metabolic genes revealed distinct clustering of bulk epithelial cells along disease severity (Fig. 7C). Pearson correlation analysis performed on ECs revealed a strong positive correlation

371 between HIF1A and key glycolytic transcripts, suggesting a hypoxia-induced glycolytic metabolic 372 reprogramming (Fig. 7D). ECs were then divided into pseudostratified ciliated and nonciliated 
subtypes based on the expression of canonical genes associated with cilia production (CFAP126, and $D N A A F)$ (Figs. 7E). The ratio of pseudostratified ciliated ECs to nonciliated epithelial cells was inversely correlated with COVID-19 disease severity (Fig. 7F). This finding suggested that SARS-CoV-2 infection produced direct injury to the ciliated EC compartment. Overexpression of glycolytic transcripts (ENO1, ADH1A3, GAPDH, ALDOA, PCK2) was noted in both ciliated and nonciliated EC subsets from COVID-19 infected patients (Fig. 7G). These results were validated by GSEA analysis, which demonstrated enrichment of glycolysis genes (Fig. 7G). We also observed decreased expression of FAO regulating genes to different extent in ciliated and nonciliated ECs from S-COVID-19 compared to HC (Fig. 7G). HIF-1A and anaerobic glycolysis gene expression was strongly correlated with reduced expression of the OXPHOS and TCA cycle genes in these EC subsets from S-COVID-19 (Fig. 7H). GSEA analysis demonstrated enrichment of glycolysis, as well as a large downregulation of OXPHOS and TCA cycle regulating genes in S-COVID-19 ciliated and nonciliated ECs (Fig. 7H). Collectively, these results suggested that oxygen deprived conditions in the COVID-19 lung mediates a metabolic switch from aerobic FAO and OXPHOS towards anaerobic glycolysis in ECs, which is strongly linked to mitochondrial dysfunction. 


\section{Discussion}

397 Metabolic syndrome and its accompanying effects are significant risk factors for COVID-19 lethality ${ }^{5,37}$. Understanding the role of cellular metabolism in COVID-19 pathogenesis has key implications in both COVID-19 prognosis and treatment. Here, we used single-cell omics techniques to construct a comprehensive metabolic landscape of immune cells involved in the SARS-CoV-2 anti-viral response. Evaluating cells from both the BALF and the blood of hospitalized COVID-19 patients revealed that lymphocyte populations undergo a global metabolic reprogramming towards anaerobic processes, resulting in compromised memory cell differentiation and effector function. We were also able to identify and define highly resolved states of dysregulated cellular metabolism in key immune cell subsets that could potentially indicate COVID-19 severity.

Despite ARDS being the main contributor to mortality and severe complications during COVID19 infection ${ }^{38}$, prior attempts to elucidate the role of metabolism in lymphopenia and immune cell exhaustion during severe COVID-19 are limited to solely analysis of patient-derived PBMCs ${ }^{8,39,40}$. Cossarizza et al. used flow cytometry and metabolic flux assays to characterize the

411 immunometabolic phenotype of isolated PBMCs from COVID-19 patients ${ }^{40}$. Although an

412 increased abundance of exhausted PD1+ lymphocytes in severe patients was found, this was not 413 accompanied by any difference in extracellular acidification (ECAR) and oxygen consumption 414 rates $(\mathrm{OXPHOS})^{40}$. Additionally, using flow cytometry and single cell RNA-sequencing, the Powell 415 group discovered a novel population of VDAC1 ${ }^{+}$exhausted T cells in PBMCs from severe COVID41619 patients ${ }^{41}$. However, they did not detect any significant change in the expression of glycolysis417 regulating genes in T cells ${ }^{41}$, which is contradictory to our results. One potential explanation for 418 this discrepancy is that the aforementioned study stimulated isolated PBMCs with $\alpha C D 3 / C D 28$ 419 polyclonal activation or with SARS-CoV-2 specific peptide libraries. Prolonged maintenance and 420 stimulation of $\mathrm{T}$ cells under normoxic conditions is not reflective of the hypoxic microenvironment 
421 conditions present during severe COVID-19 infection, which may result in the attenuation or

422 nulling of any potential metabolic differences present in COVID-19. Another potential explanation

423 arises from the fact that cellular metabolism in these studies was assessed for entire $\mathrm{T}$ cell

424 populations. Given that $\mathrm{T}$ cells are highly heterogeneous with respect to their metabolism,

425 metabolic profiles need to be assessed on specific T cell subpopulations instead of bulk T cells.

426 In contrast, by using high-dimensional flow cytometry to investigate the single-cell metabolism of

427 unstimulated PBMCs immediately after isolation, and assessing single cell transcriptomic data

428 from the BALF, we aimed to capture the metabolic state of the cells from their original

429 microenvironment. Our single-cell omics approach allowed us to detect distinct differences in

430 metabolic phenotypes of specific, highly resolved lymphocyte populations. We found that in

431 response hypoxic conditions, there is a metabolic reprogramming of CD8 and NK cell subsets

432 traditionally reliant on OXPHOS and FAO toward anaerobic glucose metabolism, which along with

433 mitochondrial dysfunction, triggers cellular exhaustion and disrupts memory differentiation,

434 resulting in a compromised anti-viral response.

435 In this study, we uncover a clear link between cellular metabolism, severe COVID-19, and 436 disrupted memory cell development. During infection, hypoxia resulting from lung epithelial cell 437 damage triggers a global metabolic reprogramming of CD8 and NK cell subsets from OXPHOS 438 and FAO towards anaerobic glucose metabolism. Upon activation, CD8 cells typically exhibit a 439 Warburg like metabolic adaptation to rely on aerobic glycolysis; thus, a metabolic switch from 440 aerobic to HIF-1a mediated anaerobic glycolysis will not impair initial T cell activation into effector 441 subsets, which is consistent with reports demonstrating hyperactivation of CD8 T cells ${ }^{42}$. 442 However, because of prolonged anaerobic glycolysis, the lung microenvironment becomes 443 increasingly hostile, resulting in nutrient depleted, hyperlactatemic, and hypoxic conditions. 444 Herein, we show that this dysregulated metabolism is heavily tied with impaired memory 445 lymphocyte formation. Although memory lymphocytes are traditionally associated with a heavy 
reliance on OXPHOS and fatty acid oxidation, we have detected multiple clusters of cellular state associated with high glucose uptake, ROS production, hypoxia mediated transcriptional

448 response, and cellular exhaustion in CD8 and NK memory cell populations that were specifically

449 enriched in hospitalized COVID-19 patients. These populations were negatively associated with

450 memory lymphocyte frequency, suggesting that this metabolic switch may halt the transition of

451 activated lymphocytes into memory cells. Pseudotime analysis and trajectory interference with

452 transcriptomic data further demonstrate differentiation of tissue-resident memory cells that was

453 tied to altered lymphocyte metabolism. In addition to anaerobic glycolysis, upregulation of

454 glutaminolysis and mitophagy was also seen in CD8 memory cells in order to sustain bioenergetic demands. Clustering solely on metabolic phenotype revealed clear distinctions between healthy, moderate, and severe patients in CD8 memory cells, suggesting the potential use of metabolic markers in predicting memory cell response.

Interestingly, we found that SARA-CoV-2 derived EC damage creates oxygen-deprived conditions in the lungs that not only induce metabolic reprogramming of various immune cell subsets, but also themselves. We found that during COVID-19 infection, differential metabolism

461 drives lung ECs towards senescence and towards acquiring a significant SASP phenotype, 462 leading to secretion of proinflammatory cytokines, reduced HLA class 2 mediated 463 immunosurveillance, and increased HLA class 1 machinery. Chronic stimulation of exhausted 464 lymphocytes, that demonstrate attenuated effector function and cytokine secretion in nutrient465 depleted microenvironments, by antigen presenting cells via HLA class 1 leads to significantly 466 increased cellular exhaustion, which further impairs the capacity of cells to differentiate into 467 memory phenotypes. Our results therefore show that the immunometabolic rewiring of ECs in the 468 BALF can be a potential mechanism for organ-specific lymphocyte exhaustion and memory cell dysfunction. Further, this observation thus highlights that unconventional antigen presentation on non-hematopoietic ECs via HLA class 1, in addition to conventional antigen presentation by 
471 professional APCs (monocyte, DC, and macrophage), can be considered as a potential target for

472 therapeutic development.

473 Reports have shown that unconventional T cells such as NKTs are attenuated in COVID-1943.

474 However, the mechanisms underlying these observations are unknown. Herein, we provided 475 evidence suggesting that ECs induce NKT exhaustion and dysfunction through prolonged antigen stimulation resulting from a hypoxia-mediated metabolic adaptation. The traditional view that NK

477 cells are short-lived innate lymphocytes is being challenged by new data demonstrating that NK 478 cells can develop long lasting, antigen- specific memory in response to viral infection ${ }^{44}$. Recently,

479 CD62 ${ }^{\text {high }}$ NK were identified as a subset possessing multiple characteristics of memory cells, demonstrating rapid responsiveness towards viral restimulation ${ }^{32}$. In the current study, we discovered that metabolic disorders cause $\mathrm{CD}_{2} 2 \mathrm{~L}^{+} \mathrm{NK}$ lymphocytopenia via impairment of memory formation. Because the abundance of GLUT- $-1^{+} \mathrm{CD} 62 \mathrm{~L}^{+} \mathrm{NK}$ cells can predict the COVID19 severity, further studies about the role of CD62 Ligh NK cells in COVID-19 pathogenesis are crucial.

485 Targeting $T$ cell glycolysis during COVID-19 infection might be a promising approach for rescuing 486 T cell fate and function. Ideally, we suggest that attempts to target T cell glycolysis in COVID-19 487 should take place after clonal expansion and formation of initial antigen specific T cells, but before 488 initiation of memory cell formation. It is possible that the efficacy of dexamethasone treatment in 489 COVID-19 patients on mechanical ventilation and supplemental oxygen ${ }^{45}$ may be due to steroid490 mediated inhibition of glycolysis ${ }^{46}$. Thus, dexamethasone administration may be able to rescue T 491 cell dysfunction, improve memory cell formation, and reduce CRS via inhibition of glycolysis ${ }^{47}$. A 492 recent stage-2 clinical trial reported that the use of 2-DG, a glucose analogue used to inhibit 493 glycolytic flux, as a therapeutic treatment for COVID-19 was highly successful in improving patient 494 outcomes, furthering glycolysis as a potential target to restore $T$ cell fate ${ }^{48}$. In support of the 495 importance of time-dependent treatment for COVID-19, type 1 IFN, which was reported to induce 
the metabolic reprogramming from glycolysis into OXPHOS and FAO in immune cells ${ }^{49}$, was only effective as a treatment option in COVID-19 when administrated early after infection ${ }^{50}$. In contrast, delayed type 1 IFN treatment resulted in worsening of COVID-19 severity due to the proinflammatory induction capacity of this cytokine ${ }^{50}$. Further, our results implicate mitophagy as a potential target for therapeutic intervention. In response to a nutrient-depleted and hypoxic microenvironment, effector CD8 T cells may upregulate mitophagy as an alternative survival

502 mechanism. However, prolonged upregulation may induce lymphocyte exhaustion and 503 mitochondrial dysfunction. Accordingly, ablating mitophagy in CTLs can potentially redirect T cells 504 towards memory cell differentiation and rescue them from exhaustion ${ }^{51}$. Furthermore, unlike glycolysis, mitophagy is not critical for $\mathrm{T}$ cell activation and effector cell differentiation ${ }^{51}$. Thus, mitophagy- targeting approaches can potentially be used immediately after COVID-19 infection.

507 Targeting mitophagy with a specific mitophagy inhibitor such as Mdivi-1 should be explored as a treatment regimen for COVID-19.

Supplemental oxygen as opposed to ventilation was shown to improve the outcome of patients with severe COVID-1952. Our current study suggests that hypoxia is a key regulator of

511 immunometabolic dysfunction during severe SARS-CoV-2 infection. Hence, efforts to maintain 512 blood oxygen saturation early in the course of infection are vital for patient recovery and 513 improvement. Immunological outcomes post- initial infection such as memory cell formation 514 dictate the severity of response upon re-exposure to the virus ${ }^{35}$. In this regard, metabolism can 515 also influence the immune response after recovery from SARS-CoV-2. Because both effector 516 function and memory differentiation are severely impaired in lymphocytes, $\mathrm{T}$ cell immunity may 517 be compromised even in recovered patients. Given the cumulatively rising COVID-19 reinfection 518 rate $^{53}$, future studies investigating how metabolism affects the humoral response, including 519 activation, antibody secretion, and long-term plasma cell differentiation of memory B, are 520 imperative. We could not detect any population of T follicular helper cells in the BALF from 13 
521 severe patients, suggesting that metabolic dysregulations also impaired germinal center

522 formation in the lungs of COVID-19 patients. In support of our current observation, hypoxia and

523 nutrient deprivation are known to suppress the generation of germinal center B cells and follicular

524 helper cells after viral infection ${ }^{54}$. Additionally, defective Tfh and germinal B cell formation in

525 spleen and lymph node, along with SARS-CoV-2-specific B cell enrichment in blood of severe

526 COVID-19 patients, were recently reported ${ }^{55}$. Overactivation of extrafollicular B cells in COVID-

52719 is also indicative of germinal center impairment ${ }^{56}$. These findings suggest that patients with

528 metabolic comorbidities or ARDS may suffer from a limited durability of antibody responses during

529 COVID-19 infection. Furthermore, knowledge of how cellular metabolism regulates memory cell

530 differentiation may help predict the reaction of patients with preexisting metabolic comorbidities

531 to vaccination. Although COVID-19 vaccines that have been approved for emergency use and

532 their short term effectiveness has been validated, their effectiveness in inducing long term

533 immunity has yet to be established ${ }^{57,58}$. Furthermore, there has yet to be an attempt to understand

534 the longevity of convalescence-induced protective immune responses in COVID-19 patients with

535 metabolic disorders.

536 Despite claims of biomarkers to predict COVID-19 severity ${ }^{59}$, no specific markers for COVID-19

537 patients with metabolic co-morbidities have been yet discovered. In the current study, using high

538 dimensional analyses, we provide a number of lowly abundant cell populations in the blood of

539 severe COVID-19 patients that can potentially predict disease severity including GLUT1 ${ }^{+}$

540 mitochondrially exhausted CTL, CD8CM, NKT and NK cells. Noticeably, a clear correlation

541 between the serum glucose level, recently identified as risk factor COVID-19 severity in patients

542 with pneumonia ${ }^{60}$, and GLUT- $1^{\text {high }} \mathrm{CD} 62 \mathrm{~L}^{+} \mathrm{NK}$ cells was observed, suggesting that the use of

543 metabolic biomarkers in combination can be strong prognostic indicator for COVID-19 disease

544 severity. 
545 Overall, our current study sheds important new light on the molecular and cellular mechanisms

546 by which immune cell metabolism regulates COVID-19 pathobiology. Shortcomings of our study

547 include a limited sample size for analysis of BALF transcriptomic data, along with a lack of

548 proteomic data for our study of lung ECs. Additionally, our assessment of cellular metabolism is

549 limited to analysis surface and intracellular metabolic marker expression, coupled with

550 transcriptomic data for key metabolic pathways. Future studies should aim to validate our results

551 using approaches that shed more light into functional metabolism such as Seahorse Metabolic

552 Flux profiling or metabolomics. Given the large diversity in the range of responses towards SARS-

553 CoV-2 infection, stratification for factors such as gender, BMI, preexisting conditions, and prior

554 treatments would help to further validate our analysis. Nonetheless, this study provides crucial

555 information about the pivotal relationship between cellular metabolism and the memory

556 lymphocyte response during severe COVID-19. Our results shed light on novel biomarkers,

557 therapeutic targets, and strategies for the COVID-19 therapy. We also provide a novel data

558 analysis pipeline for understanding single cell metabolism in an organ-specific manner. We are

559 confident that this comprehensive single-cell transcriptomic and proteomic portrait of immune cell

560 metabolism will advance COVID-19 research and help to devise novel approaches for mitigating

561 the COVID-19 pandemic. 


\section{Methodology}

\section{Sample Acquisition}

Blood from healthy donors were ordered from Research Blood Company. Blood samples from

571 hospitalized COVID-19 patients were collected from the AdventHealth hospital under protocols

572 IRB\# 1668907 and \#1590483 approved by AdventHealth IRB committee. Strict confidentiality was

573 maintained for all patients according to HIPAA confidentiality requirements. COVID-19 was

574 confirmed by PCR test at AdventHealth. Blood was used for human PBMC, plasma, and serum

575 isolation.

\section{PBMC Isolation}

577 PBMCs were isolated by density-gradient centrifugation using Ficoll. Briefly, blood specimens 578 were centrifuged at $700 \mathrm{G}$ for $7 \mathrm{~min}$ at RT for serum collection. The pellets were resuspended in 579 phosphate buffer saline (PBS). Cell suspension were carefully overlay on the top of $4 \mathrm{~mL}$ Ficoll in $58015 \mathrm{~mL}$ conical tube, followed by centrifugation at $700 \mathrm{G}$ at RT for 25 min without break. PBMCs 581 were collected from interphase between plasma and Ficoll layers. Cells were wash twice with 582 PBS to remove Ficoll residue. All the procedures were approved at $\mathrm{BSL2}^{+}$level by UCF 583 Environmental Health and Safety.

\section{Antibody Staining and Flow Cytometry}

About $5 \times 10^{5}$ cells from each sample were used for flow cytometry staining. See Table S3 for antibody information. PBMCs were first stained with live/dead in PBS for 15 min, washed with

587 FACS buffer, and stained with surface markers in FACS buffer at $4^{\circ} \mathrm{C}$ for $30 \mathrm{~min}$. Following 588 incubation, PBMCs were washed (FACS buffer, $200 \mu \mathrm{L}$ ), stained with secondary antibody mix for 589 another $15 \mathrm{~min}$, and then washed again with FACS buffer. Samples were fixed and permeabilized 590 with Fixation/Permeabilization buffer $(15 \mathrm{~min})$ and washed with FACS buffer. PBMCs were then 591 stained with the intracellular antibody staining mix at $37^{\circ} \mathrm{C}$ for $45 \mathrm{~min}$. Sample were washed once 
with Fixation/Permeabilization buffer before being resuspended in FACS buffer for flowcytometric analysis.

\section{High-dimensional Flow Cytometry Analysis}

First, the flowCore package in $\mathrm{R}$ was used to read in compensated FCS files into the $\mathrm{R}$ environment ${ }^{61}$. Automatic gating functions in openCyto were next used to filter cells for doublets

597 and debris ${ }^{62}$. Next, an arcsinh transformation with a cofactor of 5 was applied for data normalization. Data from all of the samples were then merged into one Catalyst object, upon which downstream analyses were performed ${ }^{63}$. CytoNorm was next applied to correct for batch effect between samples by aligning peaks of bimodally distributed markers ${ }^{64}$. PCA was then run

601 on bulk sample-aggregated data and the top 3 principal components were plotted. FlowSOM 602 clustering was performed on only cell surface markers used for phenotypic identification with the 603 number of expected populations set at $20^{65}$. Clusters were then annotated based upon canonical 604 marker expression. For purposes of UMAP dimensionality reduction, clusters that could not be 605 labelled were discarded before UMAP was performed. Differential abundance of cell-type 606 proportions and differential expression of MFI values were next conducted. For each identified 607 population of interest, FlowSOM was applied again on only the functional state markers with the 608 number of expected populations set at 10. Unsupervised clusters were then annotated by 609 canonical marker expression to define highly -resolved cell states for specific populations. Briefly, 610 after quality control and compensation, batch effect was corrected in makers with bimodally 611 distributed expression by the CytoNorm package in $\mathrm{R}^{64}$. UMAP and FlowSOM ${ }^{65}$ were used to 612 identify unsupervised clusters (Fig. 1H and Fig. S3A) which were assigned to specific populations 613 based on canonical marker expression (Fig. 1I and Fig. S3B). 
Single cell RNA-seq data from the BALF of 6 severe COVID patients, 3 moderate patients, and 4

617 patients as those with pneumonia experiencing respiratory distress and hypoxia and with critical

618 condition, requiring ICU care, and having been placed under mechanical ventilation, respectively.

619 Prefiltered expression matrices with UMI counts were downloaded from the GEO Database with

620 accession number GSE145926. Additionally, as suggested by the original study, data from an

621 additional BALF sample derived from a healthy donor from a separate study was used as a

622 reference ${ }^{67}$. Prefiltered expression matrices with UMI counts were downloaded from the GEO

623 Database with accession number GSE128033 and sample number GSM3660650.

\section{Data Quality Control and Preprocessing}

625 Quality control and data preprocessing was done using Seurat ${ }^{68,69}$. First, cells for which more

626 than $10 \%$ of reads were mitochondrial transcripts were discarded. Next, we removed cells that

627 had less than 1000 detected transcripts. Cells with less than 200 and greater than 6000 unique

628 genes were also filtered. Filtered data from different 14 patient samples were integrated in Seurat.

629 Individually, data from each sample was log 2 normalized and the top 2000 variable genes were

630 identified using the "vst" method in Seurat. Data from each sample was next scaled and PCA was

631 run with percentage of mitochondrial DNA and number of detected unique genes regressed out.

632 Alignment and batch effect correction was done using reciprocal PCA and canonical correlation analysis (CCA) (in accordance to standard Seurat integrated analysis workflow) on the first 30 dimensions of the data. Next, a shared nearest neighbor graph was constructed and Louvainbased optimization was run to perform unsupervised clustering. UMAP was next run on the first 30 dimensions. Data was next log 2 normalized and scaled in the "RNA" assay for expression analysis, with percentage of mitochondrial DNA and number of detected unique genes regressed 
640 SCTransform was done on the "RNA" assay to improve normalization and aid in visualization

641 purposes.

\section{T cell reintegration}

643 T cells were subsetted and split according to samples. Data from healthy control 1 and severe 1 644 were excluded from analysis due to low T cell count. To further correct for batch effect, $T$ cells

645 were then reintegrated using canonical correlation analysis in Seurat run on the first 30 646 dimensions. SCTransform was next implemented on the "RNA" assay and stored in a new "SCT" 647 assay to better normalize counts across samples for visualization purposes with percentage of 648 mitochondrial DNA regressed out. Standard log 2 normalization and scaling was then performed 649 on the "RNA" assay. Subpopulations of T-cells were next identified based upon canonical marker 650 expression.

\section{Trajectory Inference and Pseudo-temporal Ordering}

652 Monocle 3 was used to construct a trajectory upon UMAP embeddings and order cells in 653 pseudotime ${ }^{70}$. Analysis was done on both CD8 and CD4 T cells. Seurat wrapper function 654 "asMonocle" was used to create Monocle CellDataSet object from an existing Seurat object. 655 "learn_graph" function was used to construct trajectory mappings onto transferred UMAP 656 embeddings. "order_cells" was used to estimate and order cells in pseudotime. All samples for 657 CD8 and CD4 populations were ordered together and were split by disease state after ordering 658 for differential comparison of pseudotime.

\section{Metabolic Phenotype based Clustering}

660 To investigate whether metabolic phenotypes of certain cell populations alone could be used 661 alone as predictive indicators of disease severity, dimensionality reduction at both a single cell 662 and sample-wide resolution was done only on key identified differentially expressed metabolic 663 genes to see if cells/samples would cluster according to disease severity. For sample-wide 
664

665

666

667

668

669

670

671

672

673

674

675

676

677

678

679

680

681

682

683

684

685

analysis, principal component analysis was conducted and the first three principal components were visualized. For analysis at single cell resolution, UMAP was done and the first two components were visualized.

\section{Network Analysis}

For construction of gene pathway enrichment network, networkanalyst.ca was used ${ }^{71}$. All statistically significant genes were inputted along with log fold change values to construct enrichment network. Transcription factor - gene interaction network was also constructed using networkanalyst.ca ${ }^{71}$. Statistically significant genes along with log fold changes vales were inputted. The "degree" filter was first set to 100 and then the "betweenness" filter was set to 170.

\section{Downstream Analysis}

For heatmap visualizations, scaled SCTransformed values were used and the Complexheatmap package was used to generate visualization ${ }^{71}$. Hierarchical clustering and dendrogram generation were done using default settings of the package. Outliers with extremely high scaled expression values ( $>>2$ ) were set to a maximum value of 2 to not distort the rest of the Fig. . For dotplot visualizations, first a euclidean distance matrix was generated for which hierarchical clustering was then applied. Ggtree was next used for dendrogram construction ${ }^{72}$. ReactomePA package was used for functional GSEA ${ }^{73}$. All unique detected genes in the cell subset were sorted by log fold change values to create ranked list that was inputted for GSEA analysis. enrichR was used to determine over and under expressed pathways from differential expression analysis (Kuleshov) ${ }^{74}$. Corrplot package was used for generation of correlation matrices. Volcano plots were constructed using EnhancedVolcano. Other graphical visualizations were created using ggplot2, ggpubr or plotly. All further downstream analysis was done in base R.

\section{Statistical Analysis}


687 Differential expression analysis of transcript abundance was assessed using Seurat's 688 implementation of the nonparametric Wilcoxon rank-sum test. Genes were generally defined as 689 statistically significant by Bonferroni adjusted p. values less than 0.05 and log-fold change greater 690 than 0.25. For NKT cells, non-adjusted p. value was used to define differentially expressed genes 691 due to very small sample size.

692 For comparison of cell-type proportions, nonparametric Wilcoxon rank-sum test was performed, 693 and non-adjusted p. values were used to indicate significance. For comparison of median 694 fluorescent intensity (MFI), nonparametric Wilcoxon rank-sum test was also used to evaluate 695 significance. Additionally, Pearson correlation coefficient was used to indicate strength of 696 measured correlations. Student's $t$ test was used to evaluate significance of measured correlation.

\section{Figure Legend}

\section{Graphical Summary}

699 Attack of lung ECs by SARS-CoV-2 causes pulmonary damage leading to generalized hypoxia, 700 which in turn increases anaerobic glycolytic metabolism. Concomitant changes of metabolic 701 processes with increased MHC class I expression in lung ECs (ECs) drives immune cell 702 exhaustion in CD8 and NK cells. An accompanying effect is interference with immune memory 703 cell formation, which increases the vulnerability of the host to reinfection by SARS-CoV-2.

704 Therefore, protecting immune cell function in the face of SARS-CoV-2 infection by targeting 705 dysregulated immunometabolism is a promising approach to treat COVID-19 patients and 706 improve clinical outcome.

707 Fig. 1. Distinct immunophenotype of BALFs and PBPMCs from COVID-19 patients. A.

708 Schematic illustrating experimental design for multiparametric flosw cytometry and single-cell 709 RNA sequencing re-analysis. High-dimensional flow cytometry of freshly isolated PBMCs from 8 710 Healthy Control and 20 hospitalized COIVD-19 patients (B-F). B. 3-D PCA analysis conducted 
711 using bulk expression of each marker per sample as input, circles were manually drawn around

712 the PCA plot to highlight distinct clustering. C. Contour plot of kernel density for UMAP projection

713 of PBMCs, 5,000 cells from each condition (Healthy and COVID(+)) were randomly subsetted for

714 density analysis; D. UMAP projection of labelled PBMC populations from Healthy (8) and COVID-

71519 (20) patients; E. UMAP projections of PBMCs overlayed with scaled expression of markers

716 used for unsupervised clustering; F. Box-plot of cell-type proportion for each disease state, dot

717 represents individual sample, 2-sided Wilcoxon Mann Whitney test was performed to indicate

718 statistical significance; Immunophenotyping of scRNA-seq data of isolated BALFs derived from

719 healthy control, moderate and severe COVID-19 patients (F-J); G. UMAP projection displaying

720 unsupervised clusters of 66,452 cells from Healthy Control (4), Moderate (3), Severe (6) patients;

721 H. UMAP projection of labelled BALF populations annotated based upon canonical marker

expression; I. UMAP projection displaying unsupervised clusters of 7601 reintegrated T cells from Mann Whitney test was performed to indicate statistical significance.

Fig. 2. Impaired memory differentiation of CD8 T-cells in COVID-19. Pseudotime and trajectory inference analysis was conducted to evaluate the differentiation kinetics of CD8 T-cells in the BALF during SARS-CoV-2 infection. A. UMAP projection of 3,694 CD8 cells from all reintegrated samples, healthy samples alone, moderate samples alone, and severe samples alone, with trajectory mappings colored by pseudotime; B. Dot plot showing pseudotime values

732 for CD8 cells from all reintegrated samples, healthy samples alone, moderate samples alone, and severe samples alone, each dot represents a cell; C. Bar graph displaying frequency of CD8 
Fig. 3. CD8 Memory Cells in the BALF undergo anaerobic metabolic reprogramming. Analysis of metabolic phenotype of CD8 memory cells from the BALF of healthy and severe COVID-19 patients; A. Heatmap displaying expression of key differentially expressed metabolic genes for CD8 memory cells; B. GSEA enrichment plots for "Glycolysis" comparing severe vs healthy control patients, adjacent is a dotplot demonstrating expression and hierarchical clustering of select key glycolytic genes; C. GSEA enrichment plots for "TCA and Respiratory Electron Transport" pathways comparing severe vs healthy control patients, adjacent is a dotplot demonstrating expression and hierarchical clustering of select key genes involved in mitochondrial metabolism; D. Linear regression and Pearson correlation analysis between module scores for Glycolysis and Exhaustion, and module scores for glycolysis and fatty acid differentially expressed metabolic genes; F. Correlation matrix showing pearson correlation between differentially expressed metabolic genes

Fig. 4. Circulating CD8 memory cells exhibit metabolic driven exhausted phenotype in

COVID-19. Metabolic state and cell function of CD8 memory cells from Healthy and COVID-19 PBMCs were evaluated. A. Box-plot of cell-type proportion of GLUT1+ CD8 memory cells for each disease state, each dot represents individual sample, 2-sided Wilcoxon Mann Whitney test was

B. Density plot displaying distribution of ROS expression in GLUT1+ CD8 memory cells, adjacent to boxplot displaying MFI values of ROS expression in GLUT1+ CD8 memory cells for both Healthy and COVID-19 patients; C. Density 756 plot displaying distribution of LAG-3 expression in GLUT1+ CD8 memory cells, adjacent to 757 boxplot displaying MFI values of LAG-3 expression in GLUT1+ CD8 memory cells for both Healthy and COVID-19 patients; D. Box-plot of cell-type proportion of ROS+ CD8 memory cells for each disease state; E. Density plot displaying distribution of HIF-1a expression in ROS+ CD8 memory cells, adjacent to boxplot displaying MFI values of HIF-1a expression in ROS+ CD8 memory cells 
761 for both Healthy and COVID-19 patients; F. Density plot displaying distribution of VDAC1

762 expression in ROS+ CD8 memory cells, adjacent to boxplot displaying MFI values of VDAC1

763 expression in ROS+ CD8 memory cells for both Healthy and COVID-19 patients; G. Density plot

764 displaying distribution of HIF-1A expression in GLUT1+ Mitochondrially Exhausted CD8 memory

765 cells, adjacent to boxplot displaying MFI values of HIF-1A expression in GLUT1+ Mitochondrially

766 Exhausted CD8 memory cells for both Healthy and COVID-19 patients

767 Fig. 5. Aberrant metabolism of memory NK cells in COVID-19 patients.

768 Impaired metabolic phenotype and differentiation of memory-like CD62 ${ }^{+} \mathrm{NK}$ cells in circulation 769 during COVID-19. A. Box-plot of cell-type proportion of CD62L ${ }^{+}$NK cells for each disease state, 770 each dot represents individual sample, 2-sided Wilcoxon Mann Whitney test was performed to

771 indicate statistical significance; B. Box-plot of cell-type proportion of GLUT1 ${ }^{+} \mathrm{CD}_{2} \mathrm{LL}^{+} \mathrm{NK}$ cells for 772 each disease state; C. Scatterplot demonstrating correlation between serum glucose level and 773 frequency of $\mathrm{GLUT1} 1^{+} \mathrm{CD}_{2} \mathrm{~L}^{+}$cells in COVID-19 patients, linear regression line with error bars 774 displayed along with spearman correlation statistics; D. Density plot displaying distribution of HIF775 1a expression in GLUT1+ CD62L+ NK cells, adjacent to boxplot displaying MFI values of HIF-1a 776 expression in GLUT1+ CD62L+ NK cells for both Healthy and COVID-19 patients; E. Density plot 777 displaying distribution of ROS expression in GLUT1+ CD62L + NK cells, adjacent to boxplot 778 displaying MFI values of ROS expression in GLUT1+ CD62L+ NK cells for both Healthy and 779 COVID-19 patients; F. Density plot displaying distribution of HIF-1 $\alpha$ expression in ROS+ CD62L+ 780 NK cells, adjacent to boxplot displaying MFI values of HIF-1a expression in ROS + CD62L+ NK 781 cells for both Healthy and COVID-19 patients;

782 Fig. 6. Metabolic disorder impairs immune surveillance and inflammatory response of ECs.

783 The impact of metabolic reprogramming on EC immunological response was investigated. A. 784 Heatmap displaying expression of key differentially expressed genes regulating immune 785 signaling; B. Bar plot showing GSEA results of key statistically significant immune signaling 
pathways, $x$ axis displays number of enriched genes per pathway, bars are colored by adjusted p. value; C. GSEA enrichment plots for "HLA Class 2 Antigen Presentation”, "HLA Class 1 Antigen Presentation", "Toll-like Receptor Cascade", and “Interferon A/B Response" pathways comparing severe vs healthy control patients for pseudostratified ciliated epithelial subset;

D. Linear regression and pearson correlation analysis between module scores for glycolysis and HLA Class 2 Signaling; E. Linear regression and pearson correlation analysis between module scores for glycolysis and Type 1 interferon response; F. Linear regression and pearson correlation analysis between module scores for glycolysis and NF-kB signaling for all ECs; G. Network based display of transcription factor-gene interactions of differentially expressed genes between severe and healthy patients;

Fig. 7. Metabolic dysregulation of BALF-derived ECs in COVID-19. Evaluation and assessment of metabolic phenotypes of epithelial subtypes shed in the BALF. A UMAP projection of 3531 ECs from Healthy Control (2), Moderate (3), and Severe (6) patients; B. UMAP projection of labelled epithelial cell subpopulations; C. Bar graph showing distribution of pseudostratified ciliated ECs and nonciliated ECs across conditions;

D. Heatmap displaying expression of key differentially expressed metabolic genes; E. UMAP projection of ECs clustered solely on the expression of 42 differentially expressed metabolic genes; F. Correlation matrix showing spearman correlations between differentially expressed metabolic genes; G. Dot plots demonstrating expression and hierarchical clustering of select key glycolytic genes for ciliated 805 ECs, adjacent is a GSEA enrichment plot for "Glycolysis" comparing ciliated ECs from severe vs 806 healthy control patients; H. Dot plots demonstrating expression and hierarchical clustering of 807 select key mitochondrial metabolism genes for ciliated ECs, adjacent is a GSEA enrichment plot 808 for "TCA and Respiratory Electron Transport" comparing ciliated ECs from severe vs healthy 809 control patients; I. Dot plots demonstrating expression and hierarchical clustering of select key glycolytic genes for nonciliated ECs, adjacent is a GSEA enrichment plot for "Glycolysis" 
811 comparing nonciliated ECs from severe vs healthy control patients; J. Dot plots demonstrating

812 expression and hierarchical clustering of select key mitochondrial metabolism genes for

813 nonciliated ECs, adjacent is a GSEA enrichment plot for "TCA and Respiratory Electron

814 Transport"' comparing nonciliated ECs from severe vs healthy control patients

815 Fig. S1. Heatmap displaying canonical gene expression of genes used to annotate unsupervised clusters for all $\mathbf{6 6 , 4 5 2}$ cells after sample integration. FindAllMarkers function in Seurat was used to identify the top genes specific to each annotated cell population A. CD4, Macrophage, Peripheral Monocyte, Nonciliated Epithelium, B, CD8, mDC B. pDC, Proliferating T, Plasma, Ciliated Epithelium, Neutrophil, and Mast). A heatmap displaying the average expression of the genes for each cell subset was generated using the DoHeatmap function in

821 Seurat.

Fig. S2. Heatmap displaying canonical gene expression of genes used to annotate unsupervised clusters after $\mathbf{T}$ cell reintegration. FindAllMarkers function in Seurat was used to identify the top genes specific to each annotated T cell population. The average expression of the genes for each T-cell subset was generated using the DoHeatmap function in Seurat.

Fig. S3. Heatmap displaying canonical marker expression of phenotypic markers. For unsupervised clusters generated through flowSOM (A); For annotated populations of PBMCs (B); CTLs in PBMCs (C); CD8EMs in PBMCs (D); CD8TMs in PBMCs (E); CD8CMs in PBMCs (F); NKT cells in PBMCs $(\mathbf{G})$.

Fig. S4. Metabolic profiles of circulating CTLs in COVID-19. CTLs identified by unsupervised

831 clustering from patient PBMCs were evaluated to assess differential metabolic profiles. A. Density 832 plot displaying distribution of HIF-1a expression in bulk CD8 cells, adjacent to boxplot displaying 833 MFI values of HIF-1a expression in bulk CD8 cells for both Healthy and COVID-19 patients, each 834 dot represents individual sample, 2-sided Wilcoxon Mann Whitney test was performed to indicate 
statistical significance; B. Density plot displaying distribution of 2-NBDG expression in bulk CD8 cells, adjacent to boxplot displaying MFI values of HIF-1A expression in bulk CD8 cells for both Healthy and COVID-19 patients. C. Density plot displaying distribution of GLUT-1 expression in CTLs, adjacent to boxplot displaying MFI values of GLUT-1 expression in CTLs both Healthy and COVID-19 patients; D. Box-plot of cell-type proportion of GLUT1+ CTLs for each disease state; E. Box-plot of cell-type proportion of ROS+ CTL for each disease state; F. Density plot displaying distribution of VDAC1 expression in GLUT1+ CTLs, adjacent to boxplot displaying MFI values of VDAC1 expression in GLUT1+ CTLs for both Healthy and COVID-19 patients. G. Density plot displaying distribution of LAG-3 expression in GLUT1+ CTLs, adjacent to boxplot displaying MFI values of LAG-3 expression in GLUT1+ CTLs for both Healthy and COVID-19 patients. H. Heatmap displaying canonical expression of labelled populations from the second round of unsupervised clustering; I. Box-plot of cell-type proportion of GLUT1+ Mitochondrially Exhausted CTLs for each disease state

\section{Fig. S5. Differential metabolic phenotype of BALF derived CTLs in severe COVID-19 A.} Heatmap displaying expression of key differentially expressed metabolic genes for CTLs; B. GSEA enrichment plots for "Glycolysis" comparing severe vs healthy control patients, adjacent is a dotplot demonstrating expression and hierarchical clustering of select key glycolytic genes; $\mathbf{C}$. GSEA enrichment plots for "TCA and Respiratory Electron Transport" pathways comparing severe vs healthy control patients, adjacent is a dotplot demonstrating expression and hierarchical clustering of select key genes involved in mitochondrial metabolism; D. Violin plot demonstrating expression of NFE2L2 and PRDX2 across disease states.

Fig. S6. A. Heatmap displaying expression of key differentially expressed metabolic genes; B. GSEA enrichment plots for "Glycolysis" and "TCA and Respiratory Electron Transport" pathways comparing severe vs healthy control patients; C. Dot plots demonstrating expression and hierarchical clustering of select key metabolic genes; D. Volcano plot of differentially expressed 
860

861

862

863

864

865

866

867

868

869

870

871

872

873

874

875

876

877

878

879

880

881

882

883

884

genes between severe and healthy COVID-19 patients, $x$ axis shows log2 fold change, $y$ axis shows adj. p value

Fig. S7. A. UMAP projection of 2603 CD4 cells from all reintegrated samples, healthy samples alone, moderate samples alone, and severe samples alone, with trajectory mappings colored by pseudotime; B. Dot plot showing pseudotime values for CD4 cells from all reintegrated samples, healthy samples alone, moderate samples alone, and severe samples alone, each dot represents a cell.

Fig. S8. Metabolic profiling of NKT in PBMCs and BALF of COVID-19 patients. NKT cells detected by unsupervised clustering in PBMCs were assessed for expression of metabolic markers and cell function. A. Box-plot of cell-type proportion of GLUT1+ NKT cells for each disease state, each dot represents individual sample, 2-sided Wilcoxon Mann Whitney test was performed to indicate statistical significance; B. Box-plot of cell-type proportion of ROS+ NKT cells for each disease state, adjacent is density plot displaying distribution of HIF-1a expression in ROS+ NKT cells, alongside boxplot displaying MFI values of HIF-1a expression in ROS+ NKT cells for both Healthy and COVID-19 patients; C. Box-plot of cell-type proportion of GLUT1+ Mitochondrially Exhausted NKT cells for each disease state, adjacent is density plot displaying distribution of HIF-1a expression in GLUT1+ Mitochondrially Exhausted cells, alongside boxplot displaying MFI values of HIF-1a expression in GLUT1+ Mitochondrially Exhausted cells for both Healthy and COVID-19 patients; D. Heatmap displaying expression of key differentially expressed metabolic genes for NKT cells; E. GSEA enrichment plots for "TCA and Respiratory Electron Transport" pathways comparing severe vs healthy control patients, adjacent is a dotplot demonstrating expression and hierarchical clustering of select key genes involved in mitochondrial metabolism; F. GSEA enrichment plots for "Glycolysis" comparing severe vs healthy control patients, adjacent is a dotplot demonstrating expression and hierarchical clustering of select key glycolytic genes; G. UMAP projections of NKT cells clustered solely on 
885

886

887

888 889 corresponding author.

890

891

892

893

894

895

896

897

898

899

900

901

902

903

904

905

\section{Code Availability}

\section{Ethics Declaration} \#1668907 and \#1590483.

\section{Competing interests}

\section{Author contribution}

\section{Acknowledgements}

the expression of key differentially expressed metabolic genes; H. Correlation matrix showing pearson correlation between differentially expressed metabolic genes

The source code used to reproduce our analysis can be accessed upon request from the

This study was approved by the IRB committee of AdventHealth hospital and UCF under protocols

The authors declare no conflicts of interest.

H.N. conceived the research project. S.G. and H.N performed data analysis, drafted manuscript and wrote the paper. HN, SG, STG, DL, NTL contributed to revise the manuscript. HN, SG, AA, $\mathrm{KE}, \mathrm{DL}, \mathrm{TH}, \mathrm{SA}$, and AM contributed to the experimental process.

The current study is conducted with the support from the University of Central Florida start-up funding and College of Medicine, University of Central Florida COVID-19 seed funding support to H.N. We would like to thank Drs. Alessandro Sette and Daniela Weiskopf at La Jolla Institute for Immunology for sharing COVID-19 peptide mix and Dr. Justine Tigno-Aranjuez for sharing flow antibody. Reagents and protocol for detection of COVID-19 antigen by ELISA were obtained through BEI Resources, NIAID, NIH. 
Fig. 1
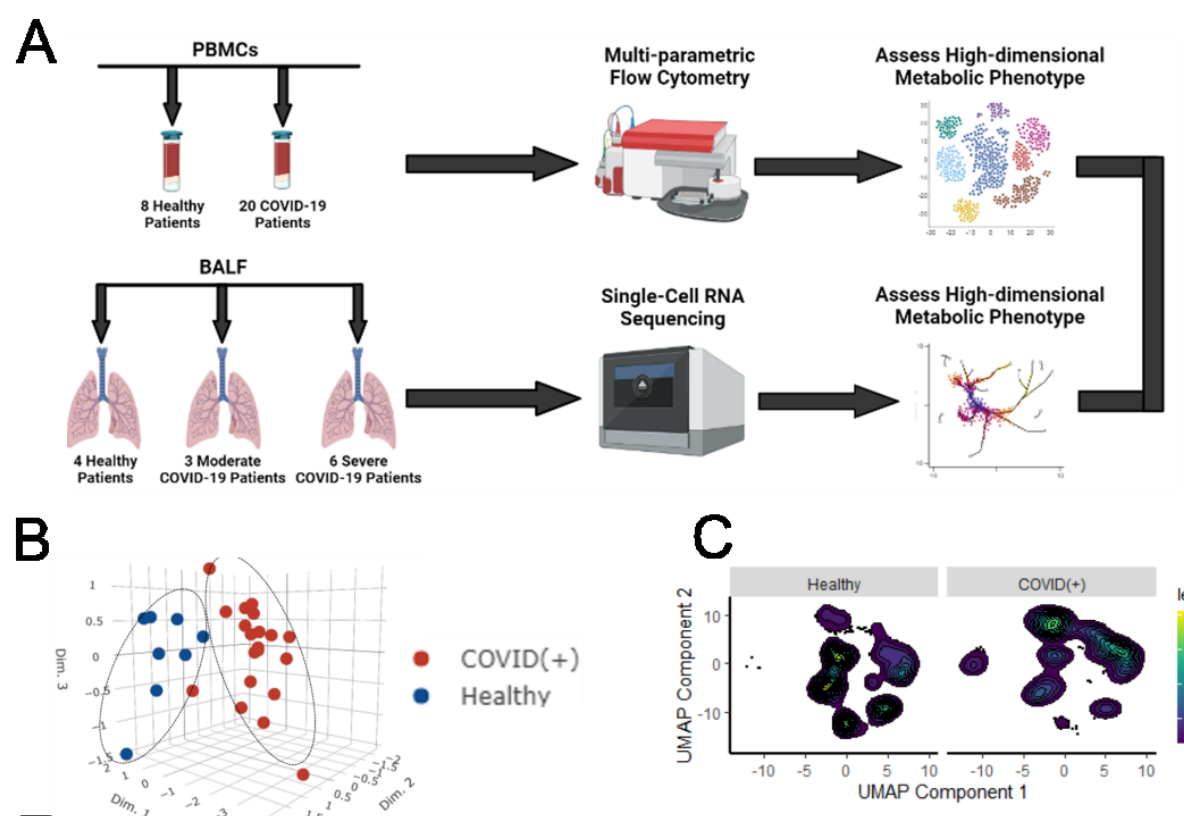

E
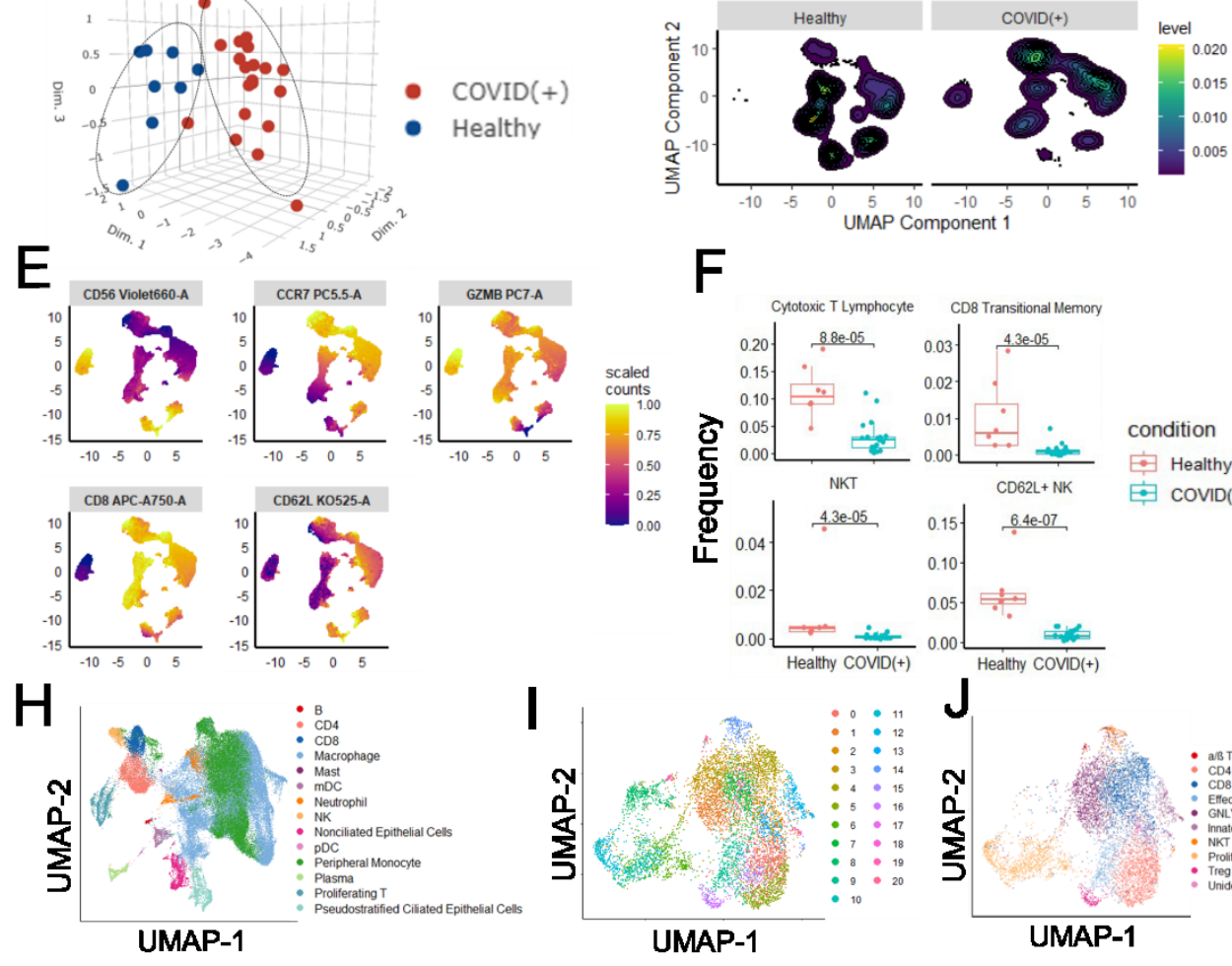

D

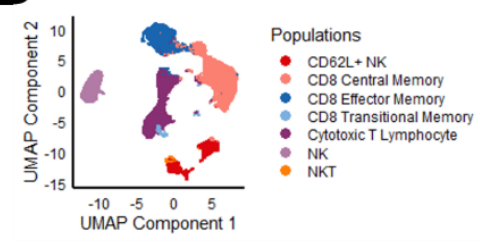

F

G

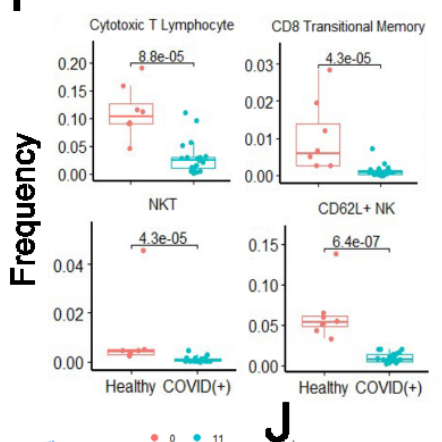

condition Healthy .

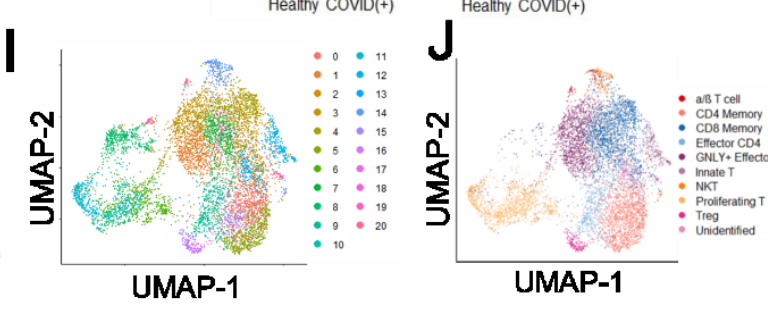

K CD8 Memory NKT

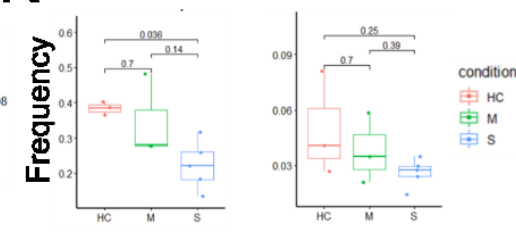


Fig. 2

A

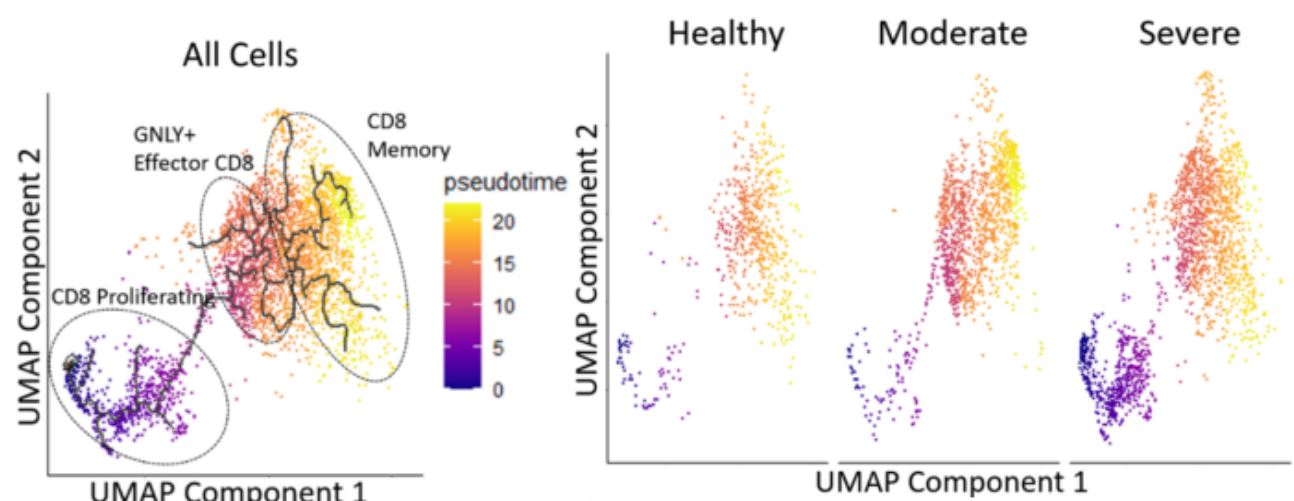

B

UMAP Component 1
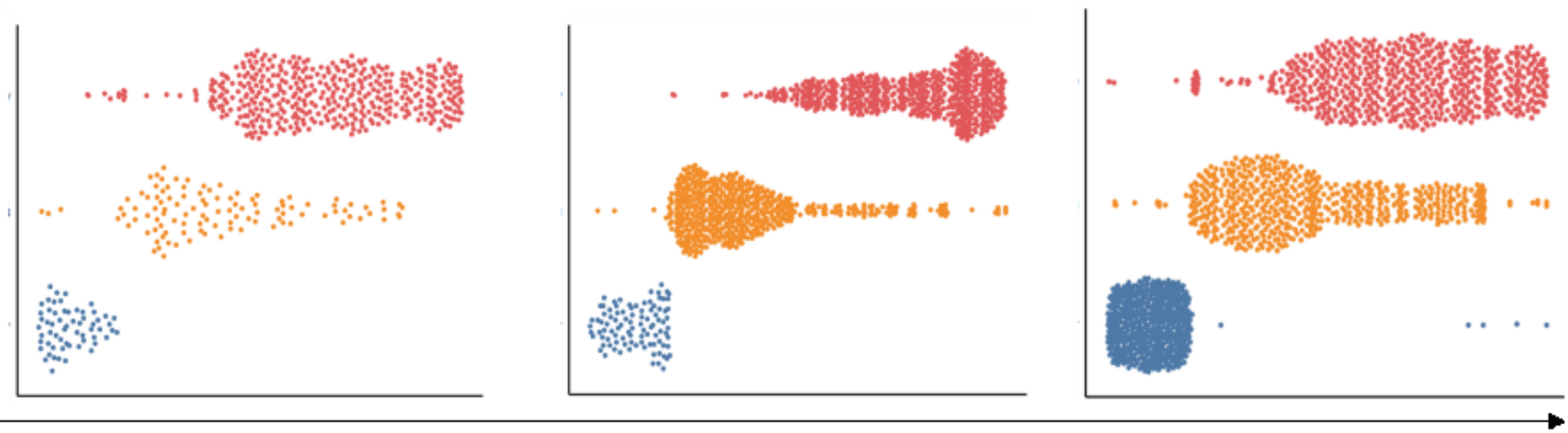

C

Preudotime
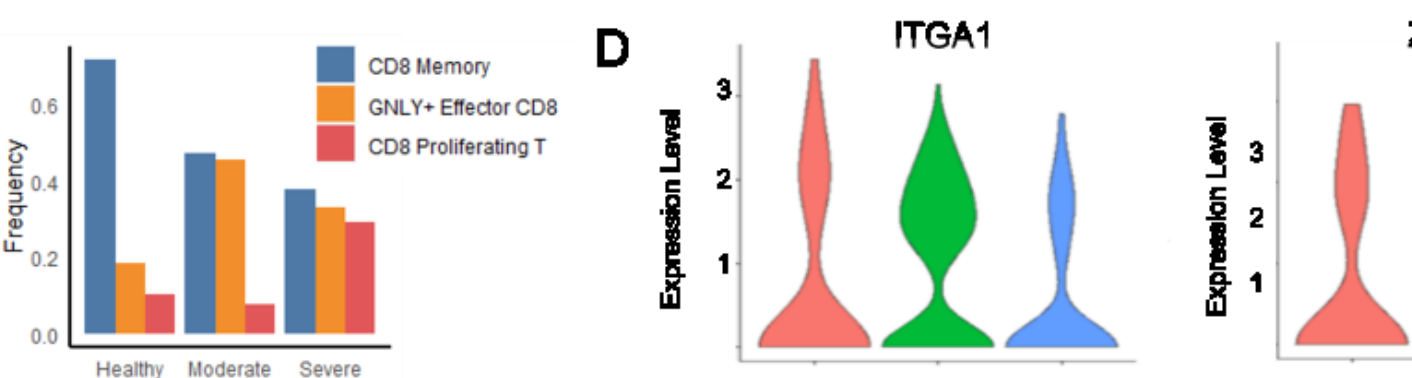

ZNF683

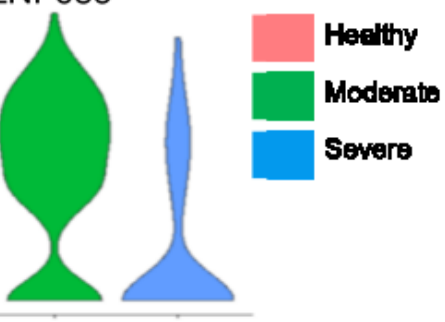


Fig. 3
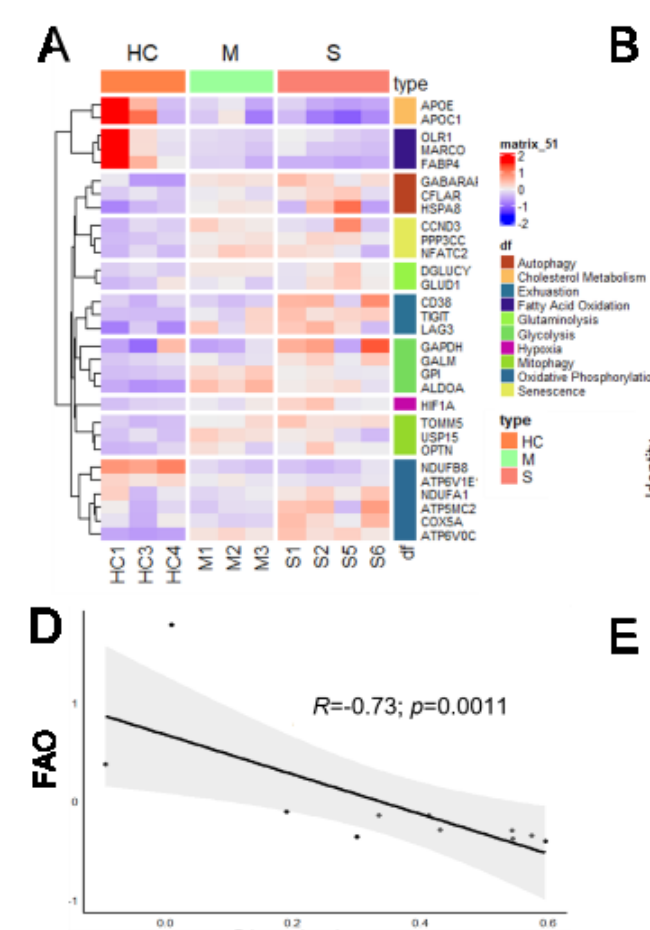

Glycolysis

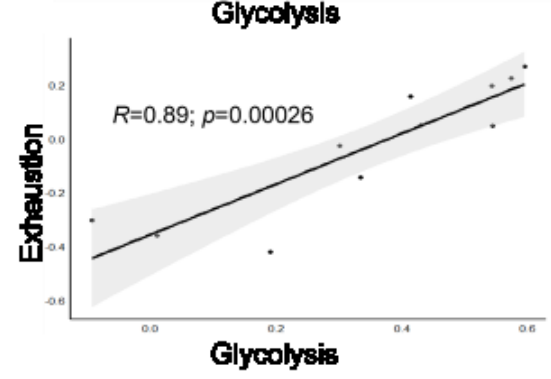

B
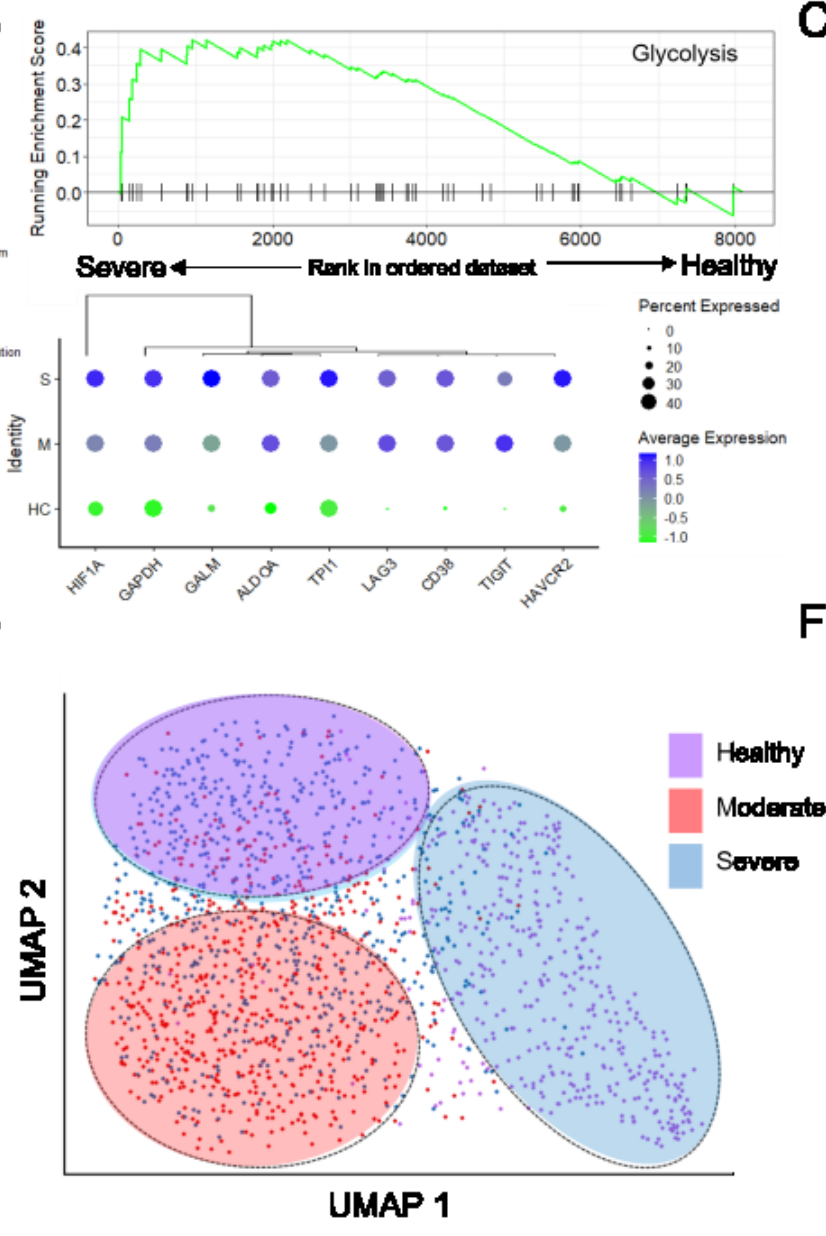

$\mathrm{C}_{\mathrm{\circ}}$
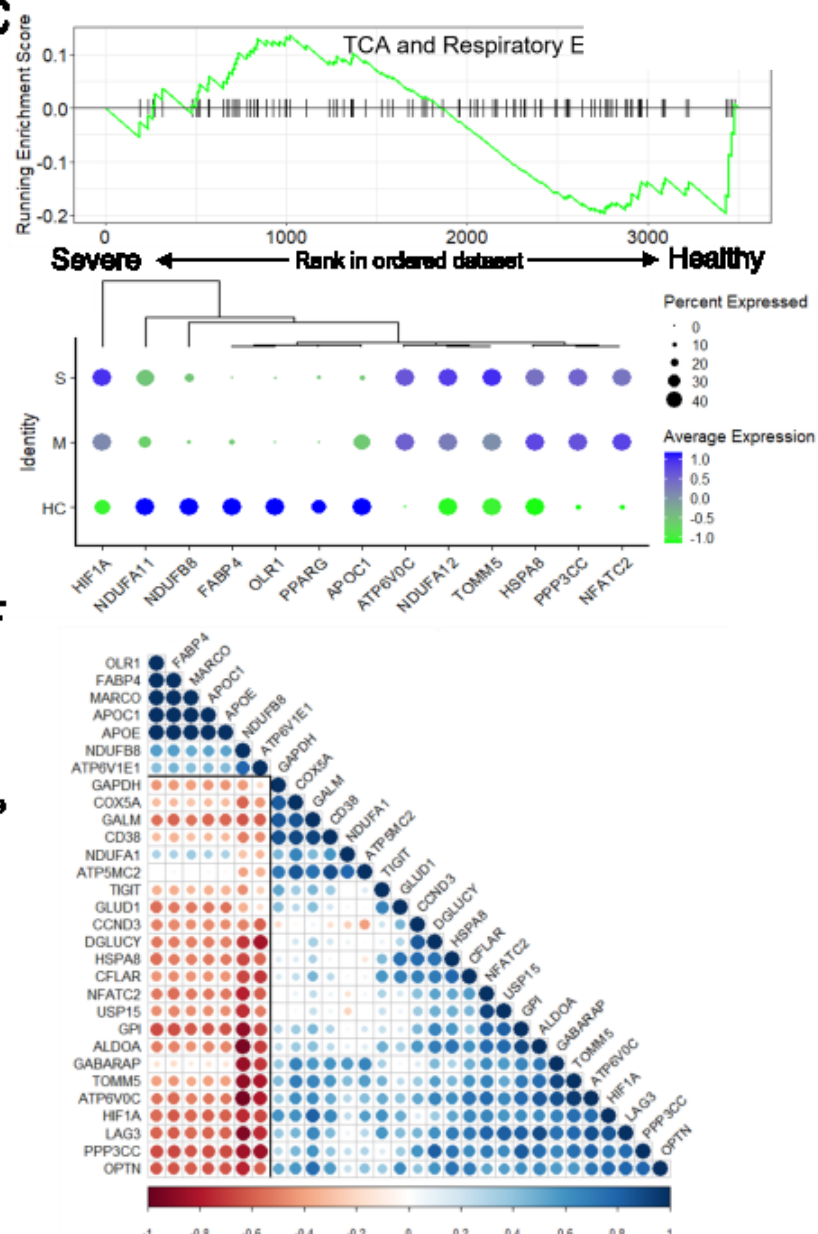

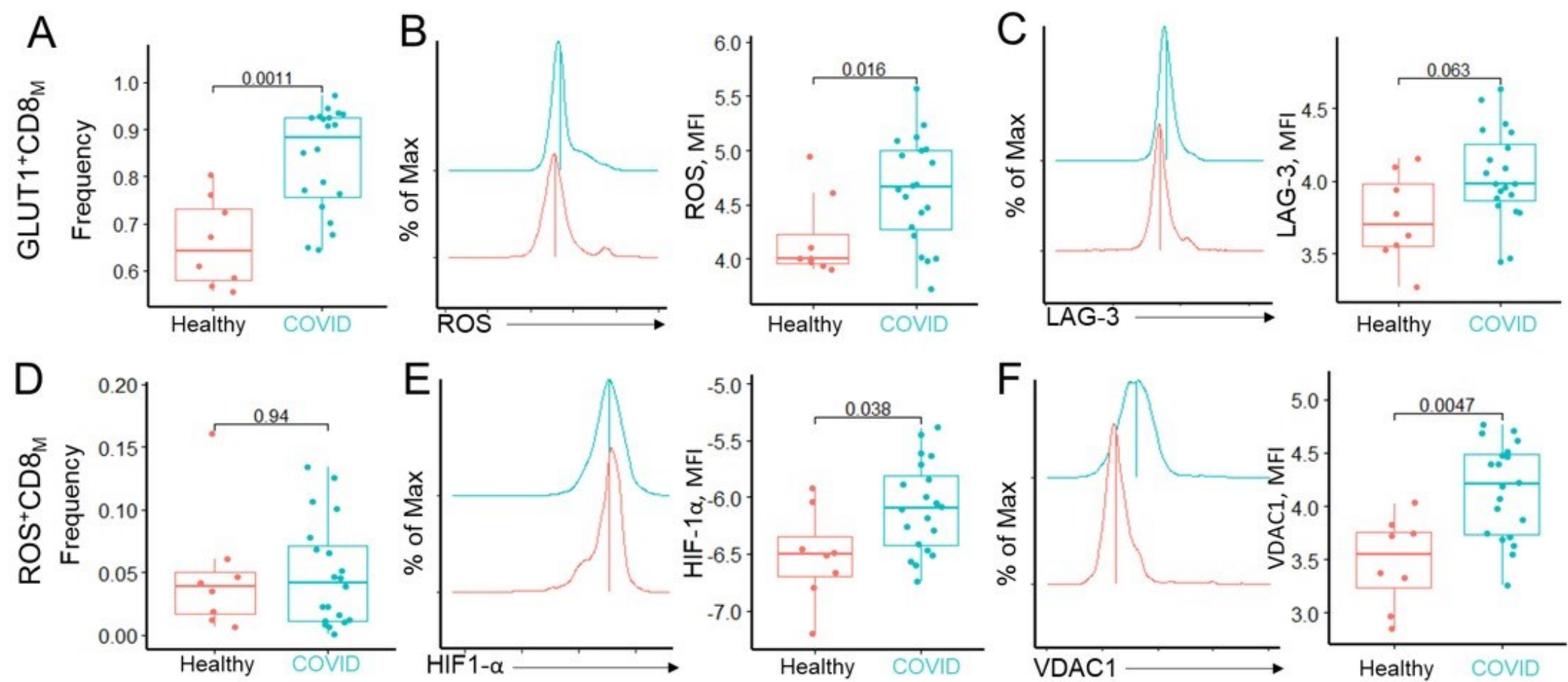

G GLUT1+ Mitochondrially Exhausted $\mathrm{CD} 8_{\mathrm{M}}$

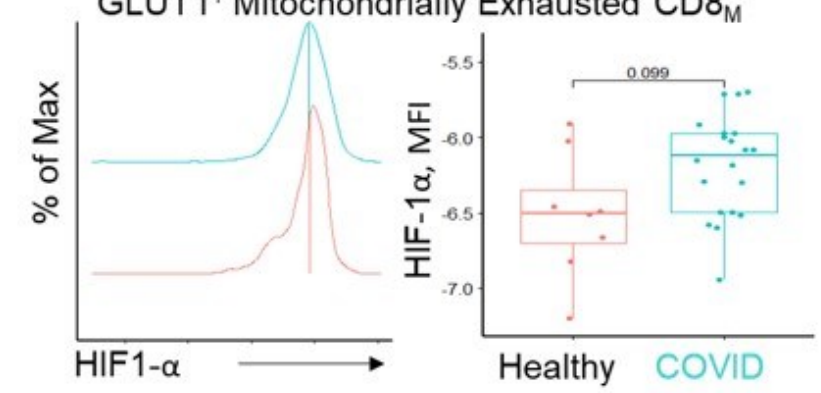


Fig. 5
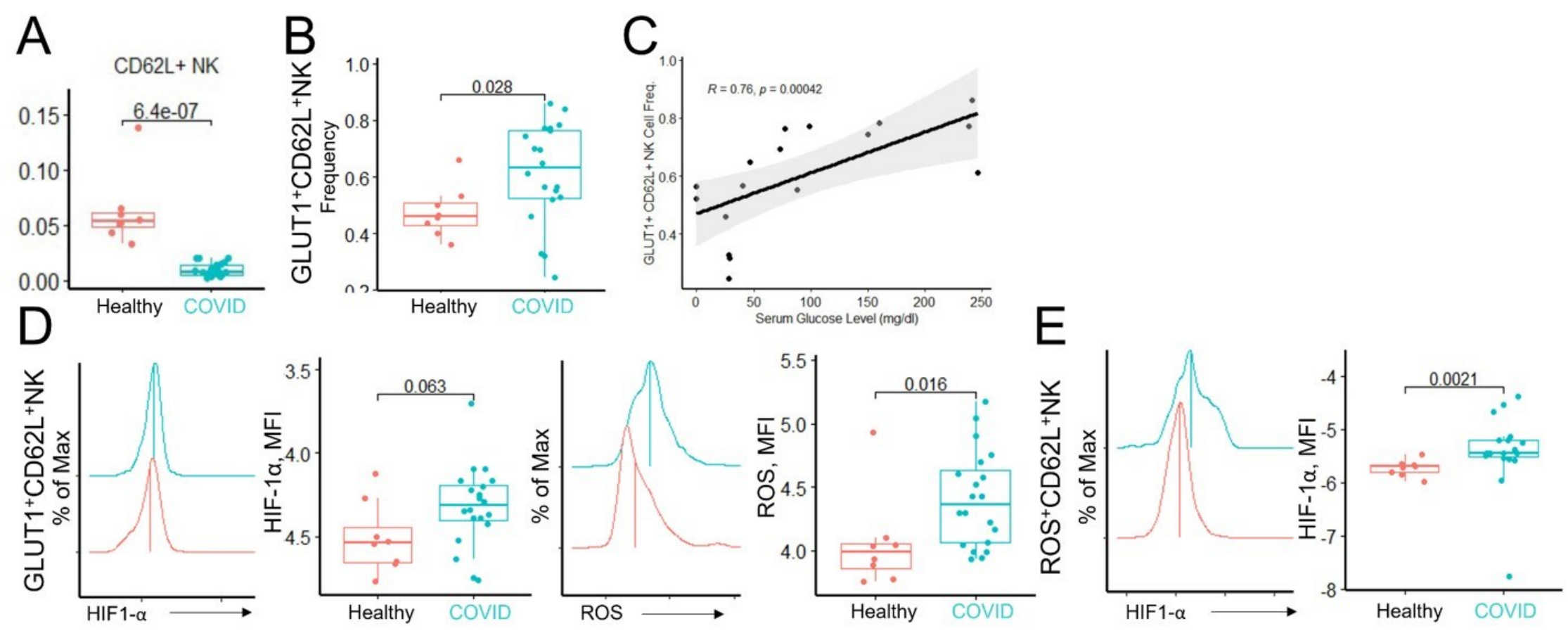

918

919

920

921 
Fig. 6
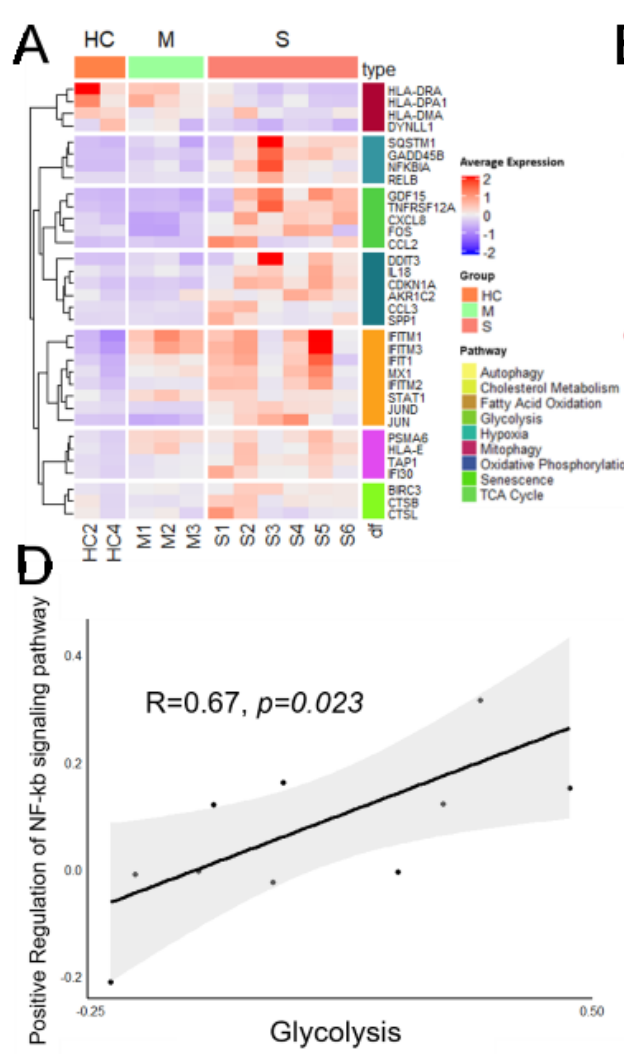

$\mathbf{F}$
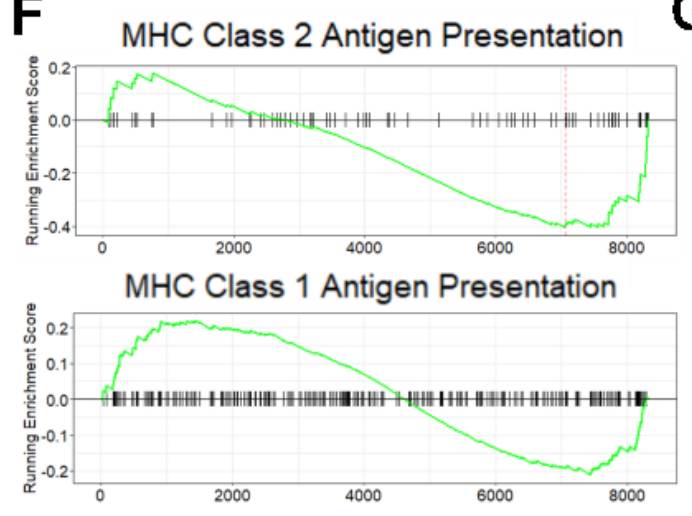

Severe $\longleftarrow$ Rank in ordered dataset $\longrightarrow$ Healthy

D

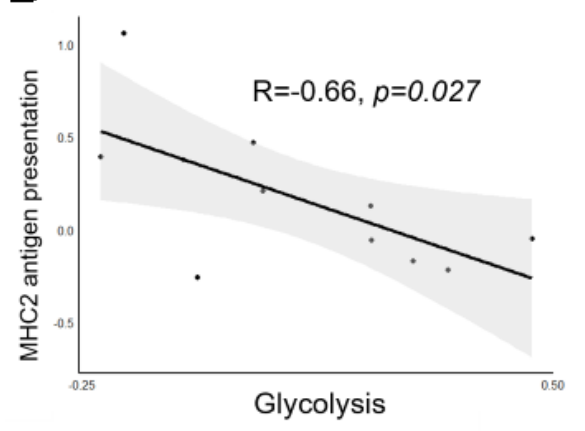

B C

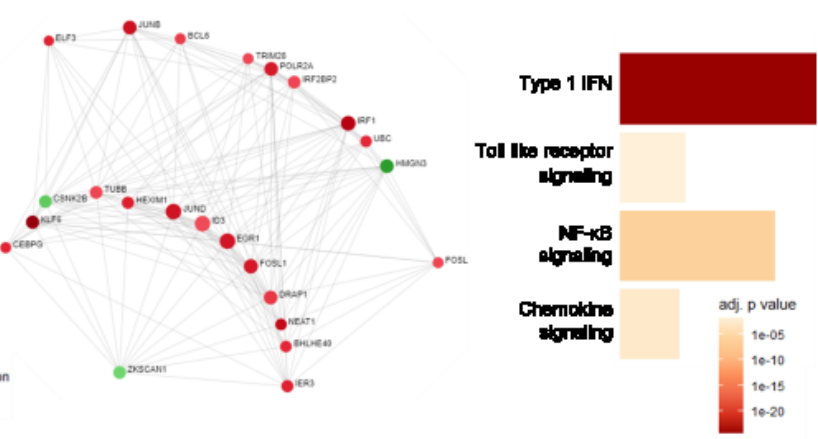

E

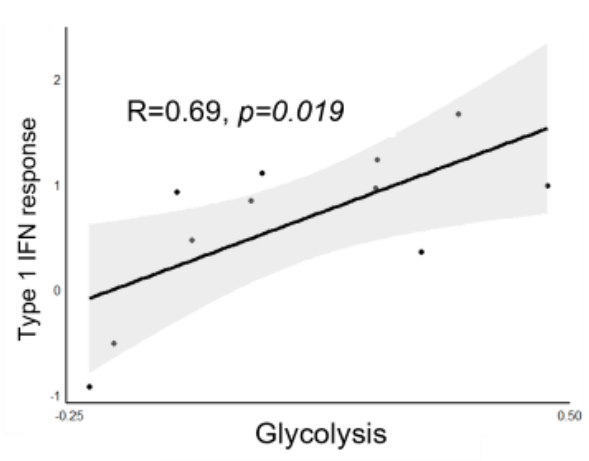

$\mathbf{G}$
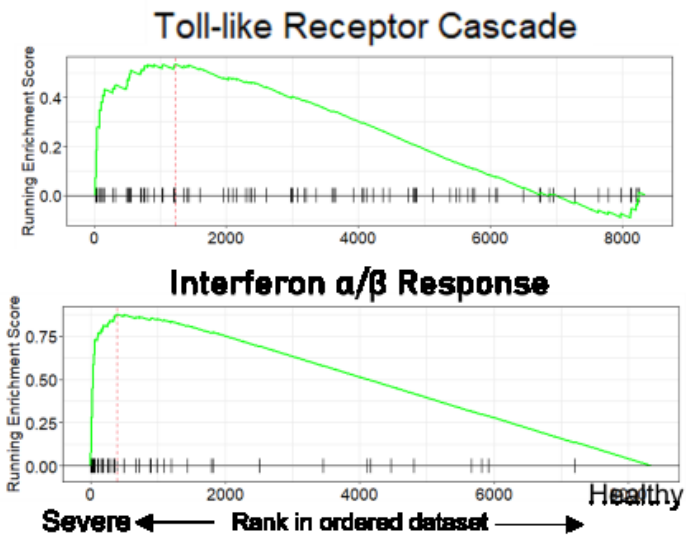
bioRxiv preprint doi: https://doi.org/10.1101/2021.12.06.471421; this version posted December 8, 2021. The copyright holder for this preprint (which was not certified by peer review) is the author/funder. All rights reserved. No reuse allowed without permission.

Fig. 7
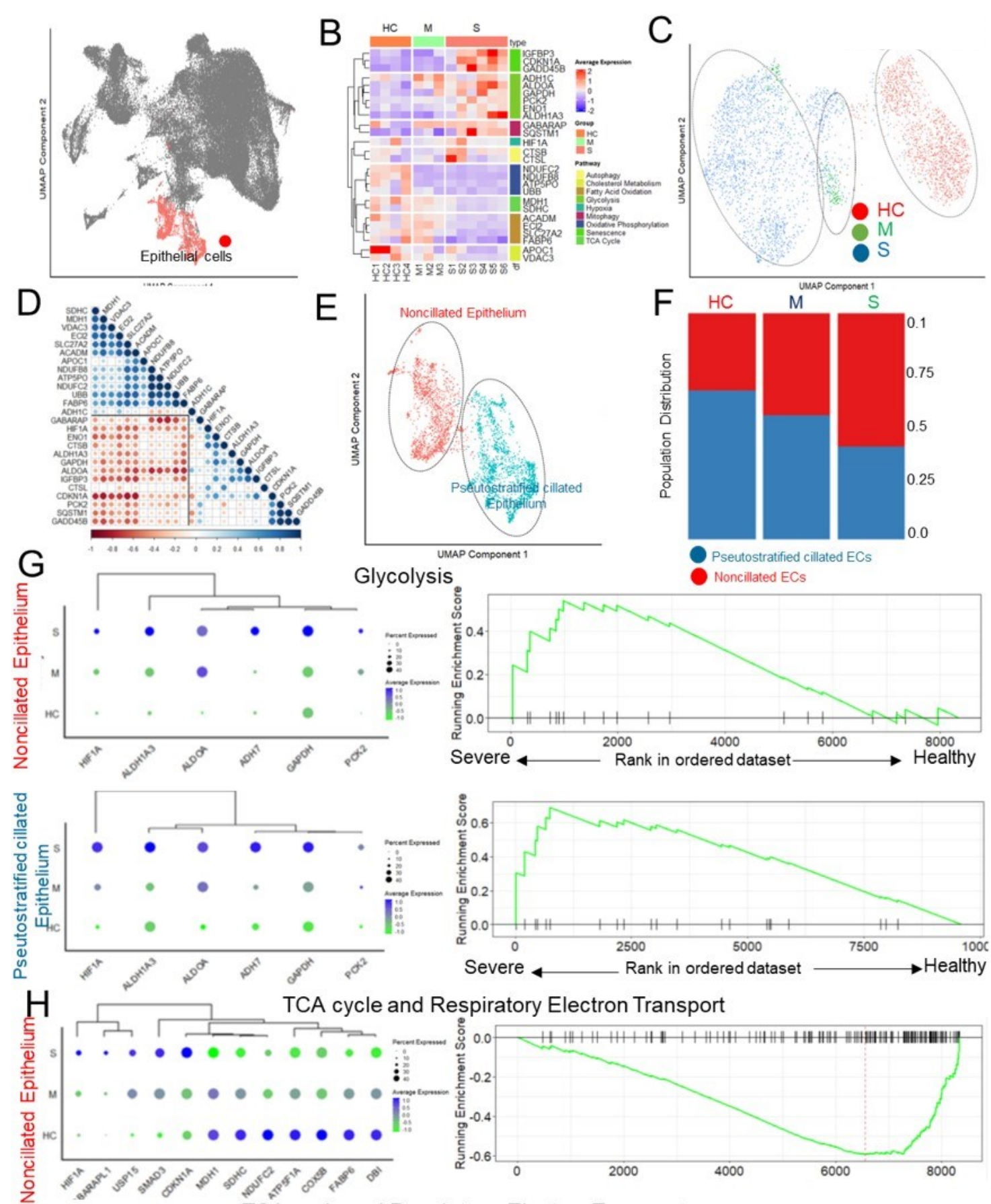

TCA cycle and Respiratory Electron Transport

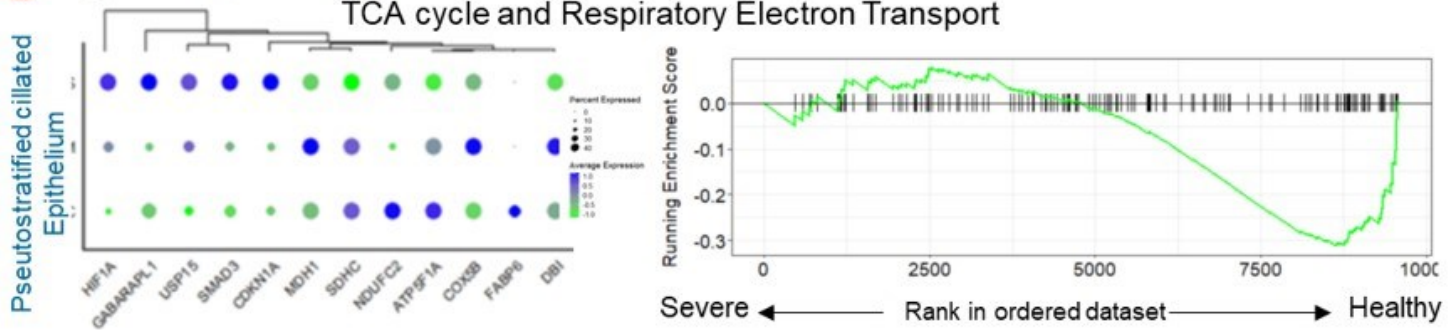


bioRxiv preprint doi: https://doi.org/10.1101/2021.12.06.471421; this version posted December 8, 2021. The copyright holder for this preprint (which was not certified by peer review) is the author/funder. All rights reserved. No reuse allowed without permission.

Fig. $\mathbf{S 1}$

A

CD4 Macrophage CD8 Peripheral Nonciliated Monocyte Epithelium

B

CD8

$\mathrm{mDC}$

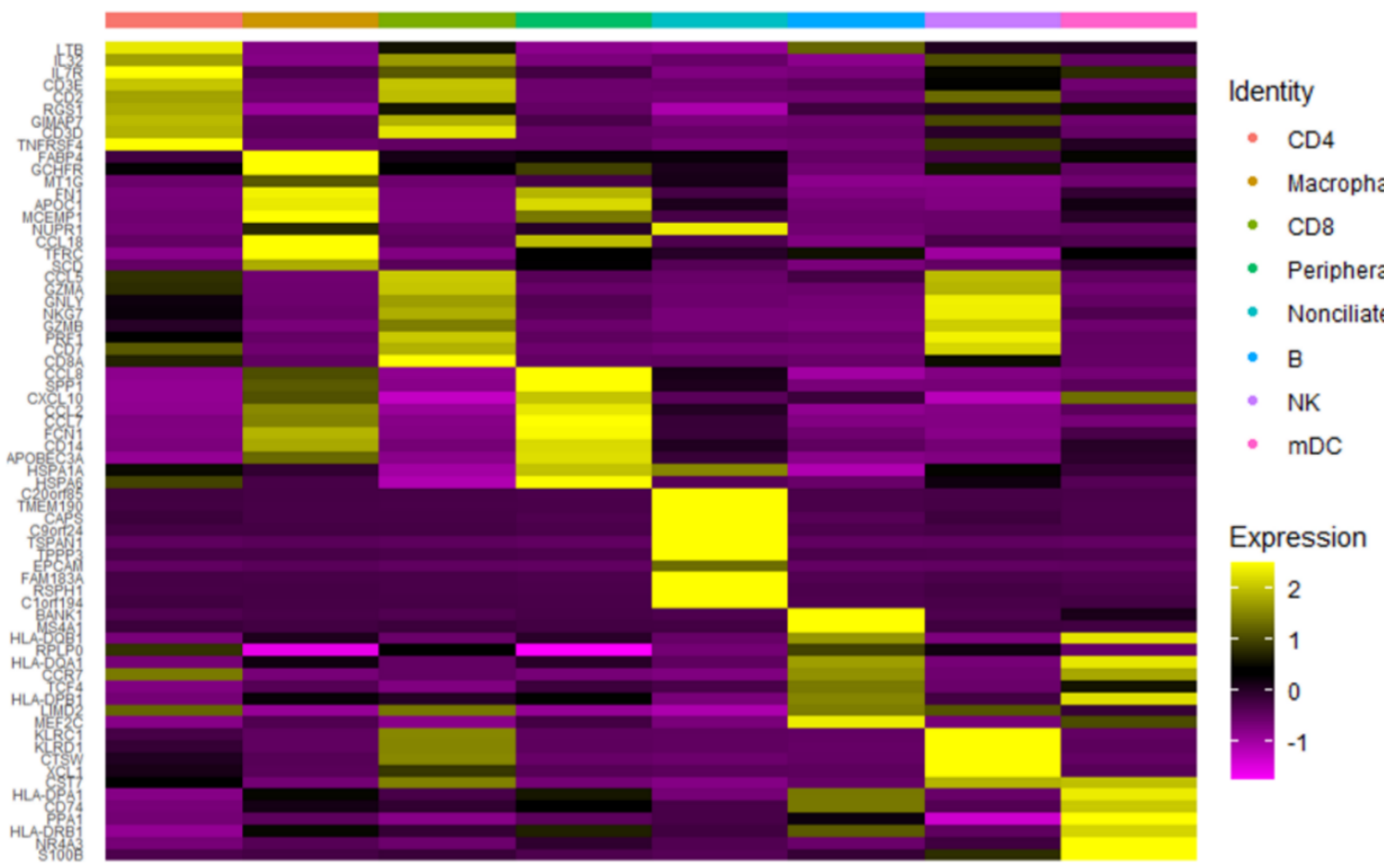

B

$\begin{array}{lcccc}\text { pDC } & \text { Proliferating } & \text { Plasma } & \begin{array}{c}\text { Ciliated } \\ \text { Epithelium }\end{array} & \text { Neutrophil }\end{array}$
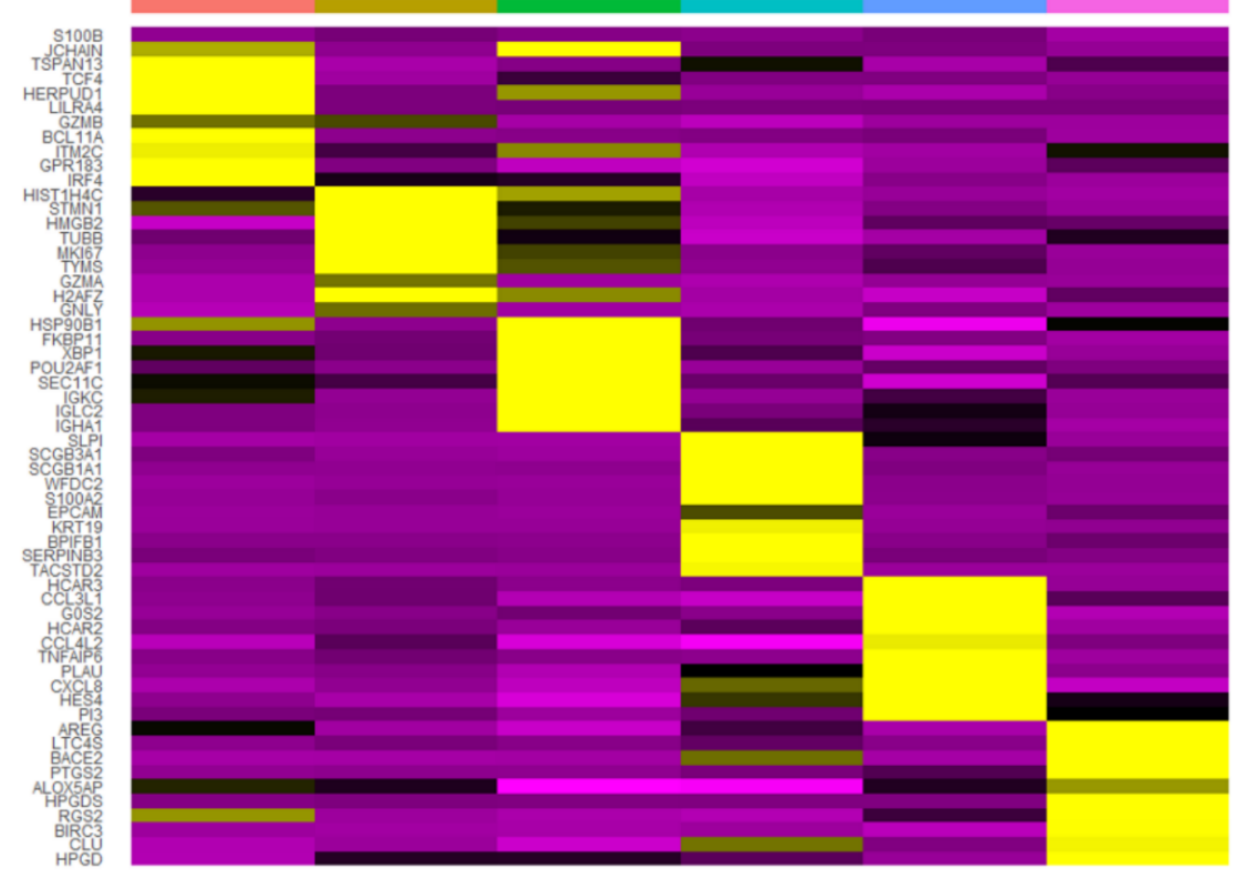

Identity

- pDC

- Proliferating_T

- Plasma

- Pseudostratified_Ciliated_Epithelium

- Neutrophil

- Mast

\section{Expression}

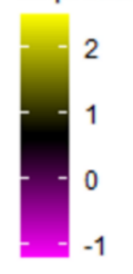


Fig. 52

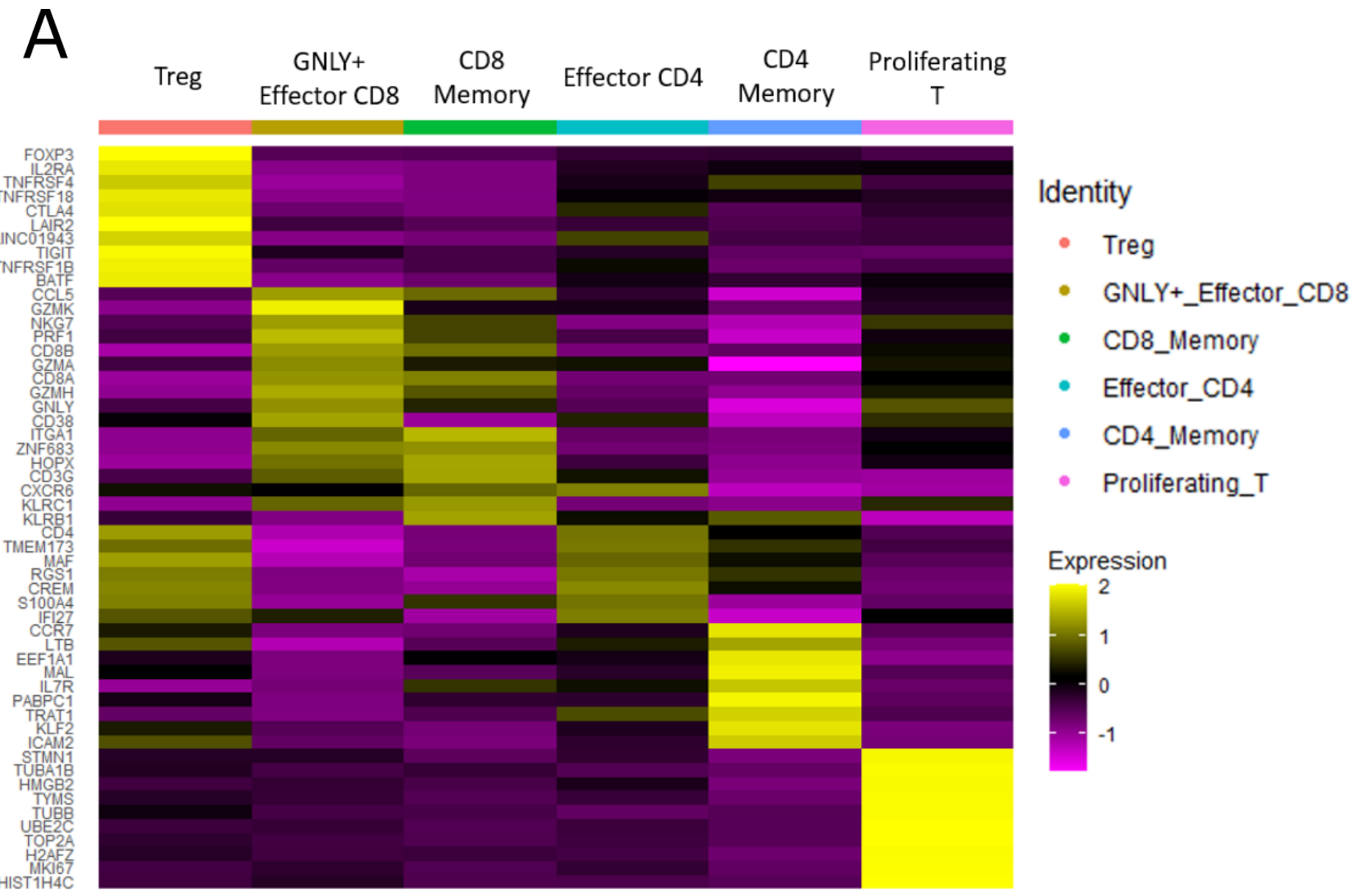




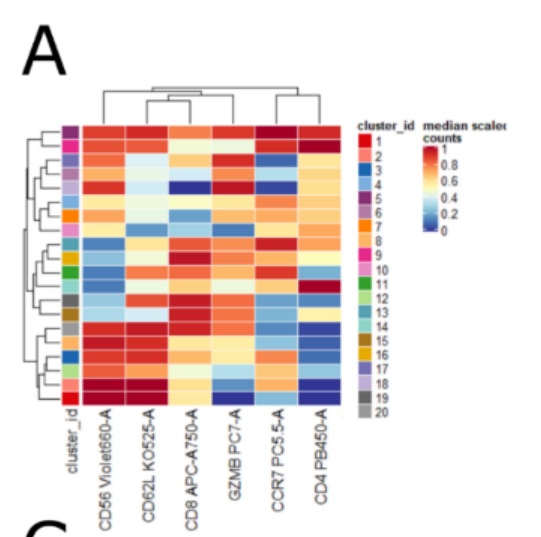

B

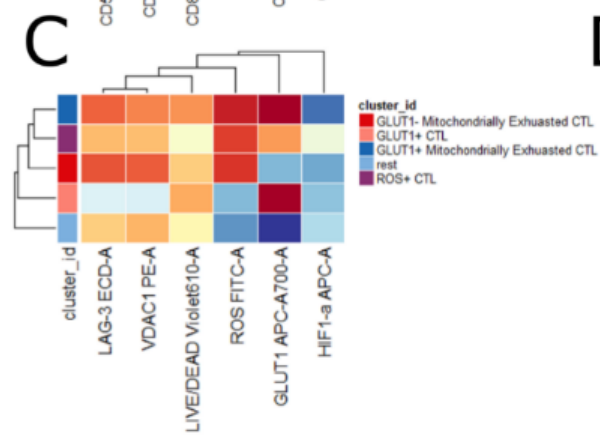

$D$ E

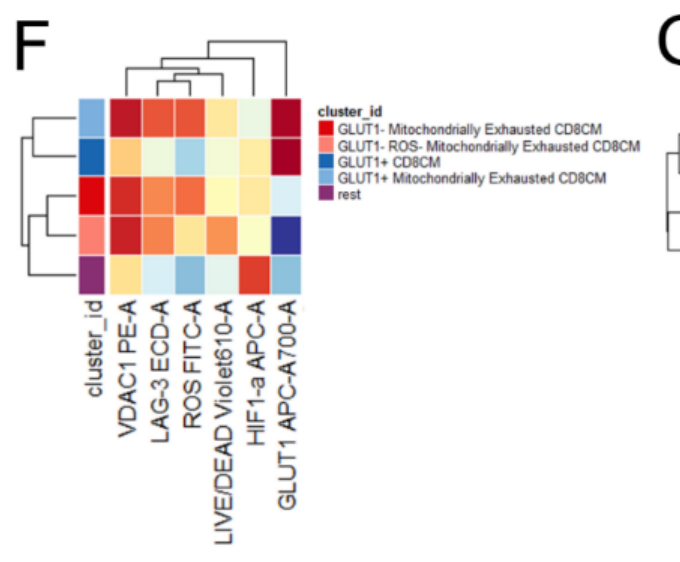

G
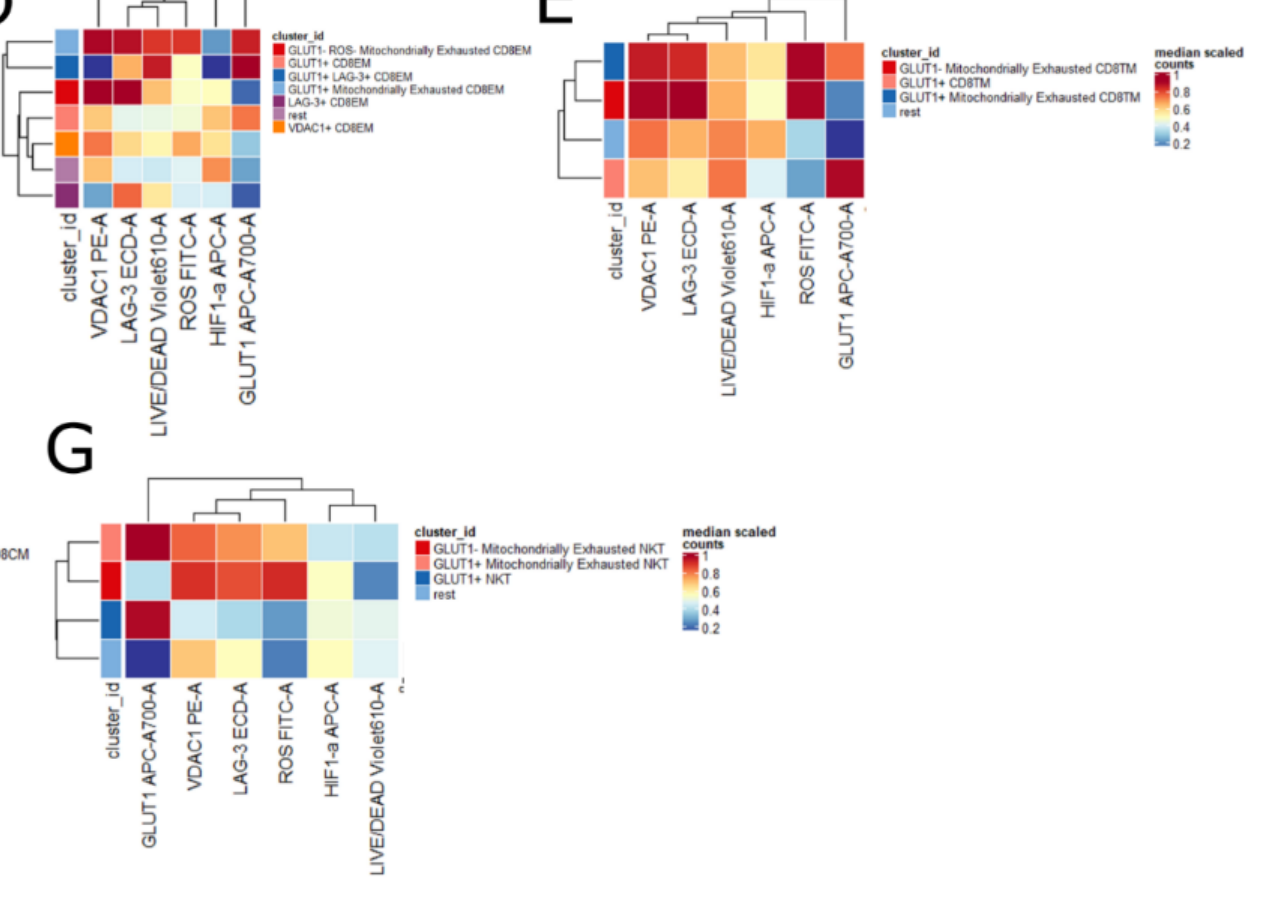
A

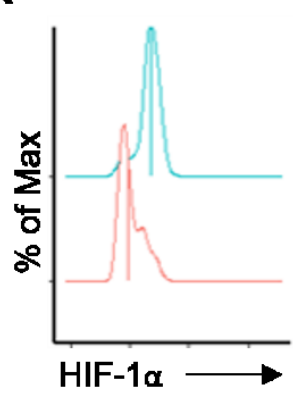

CD8

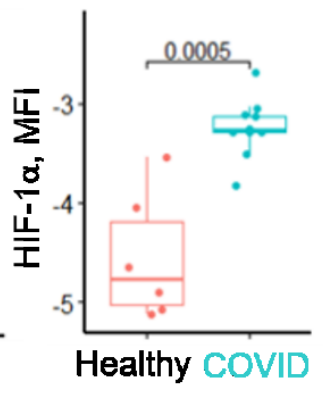

C

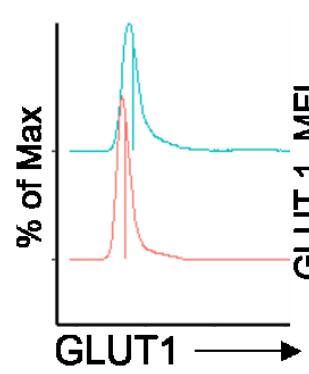

CTL

$\mathrm{F}$

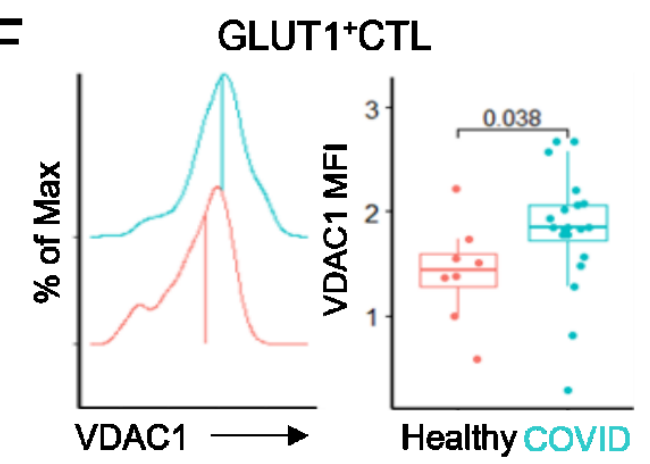

$\mathrm{H}$

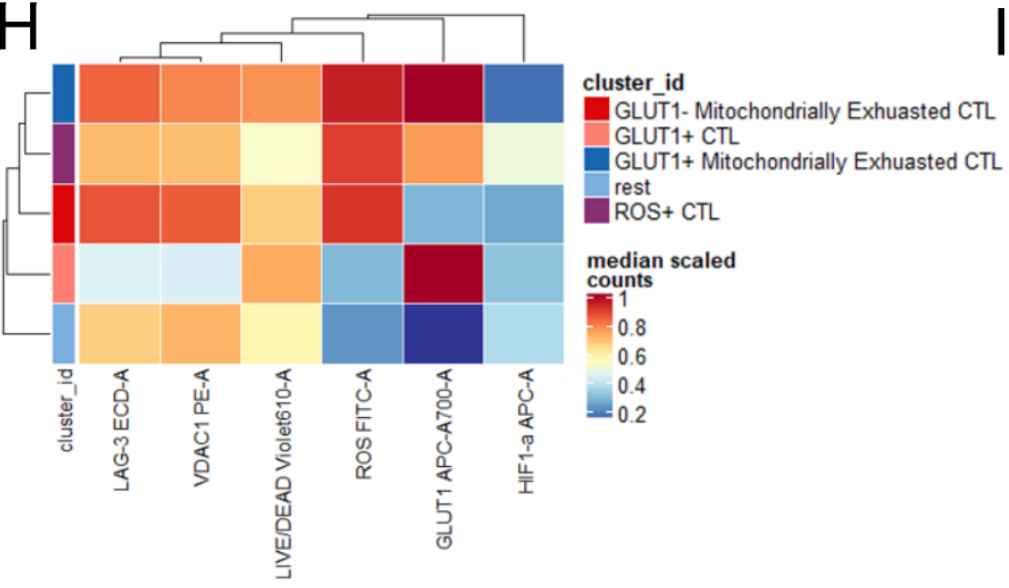

B $\quad$ CD8
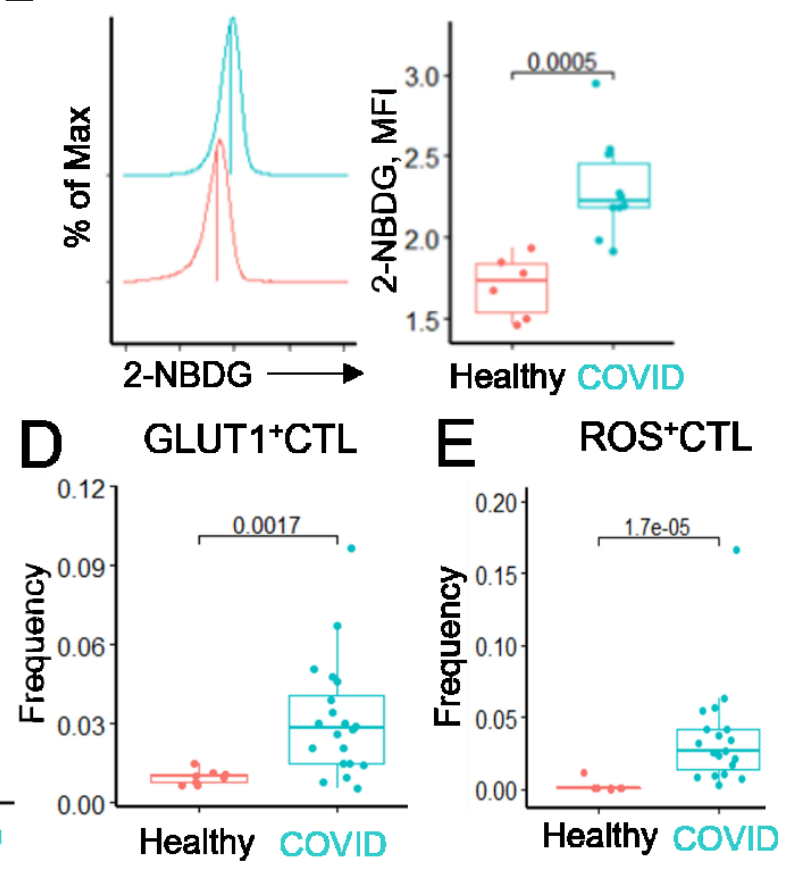

G

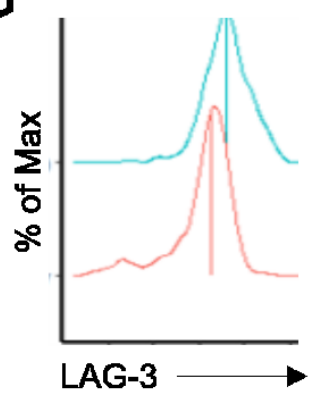

GLUT1+CTL

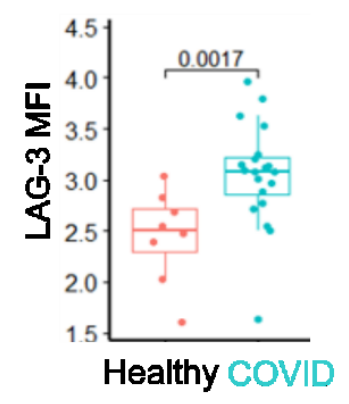

GLUT1 ${ }^{+}$Mitochondrially Exhausted CTL

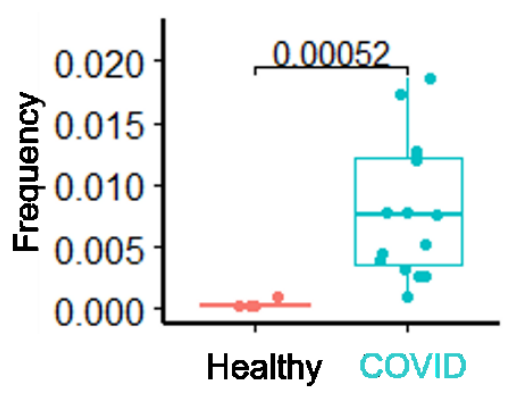



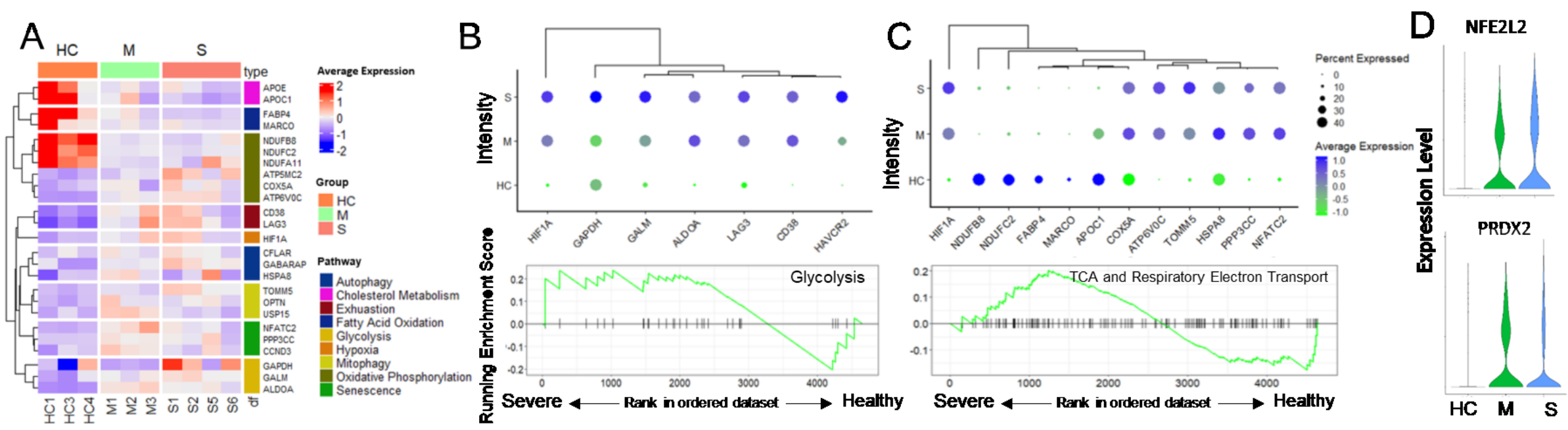


\section{$937 \quad$ Fig. $\mathbf{S 6}$}

Proliferating T Metabolism
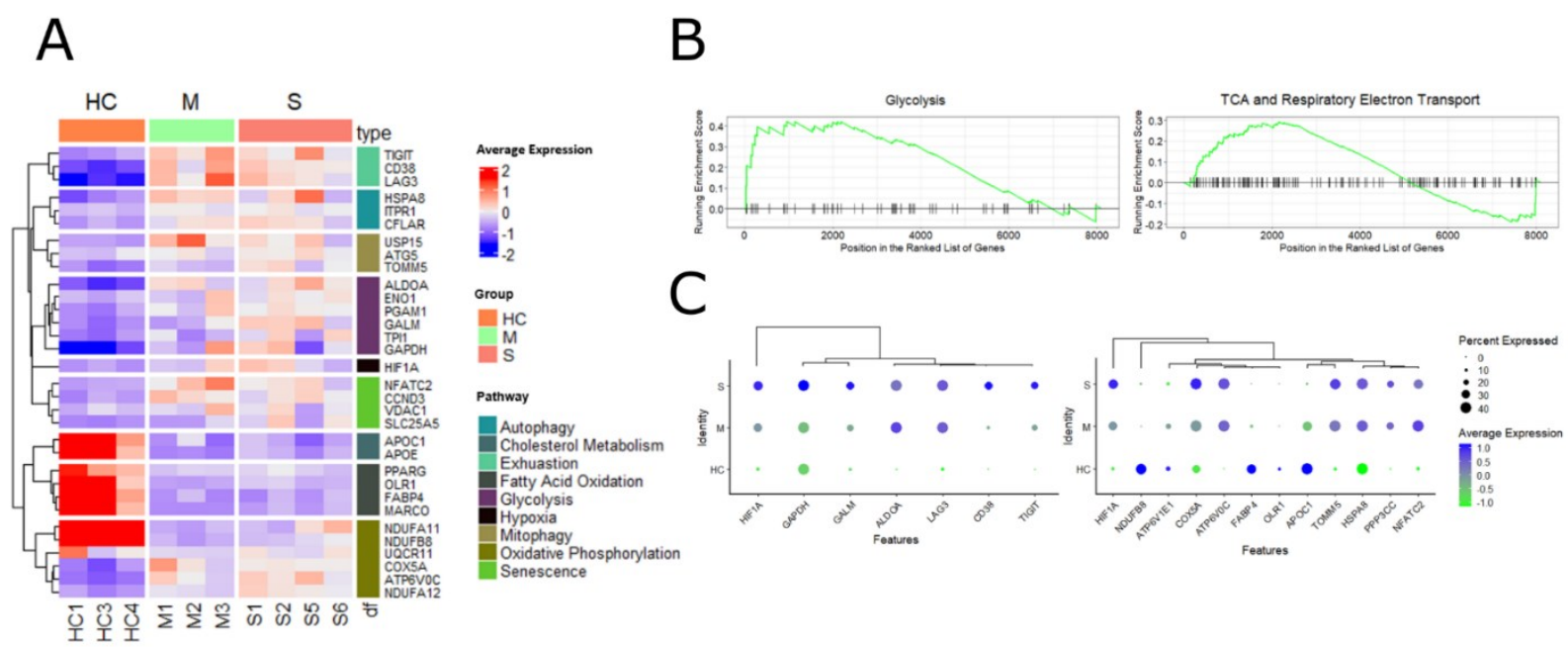

C

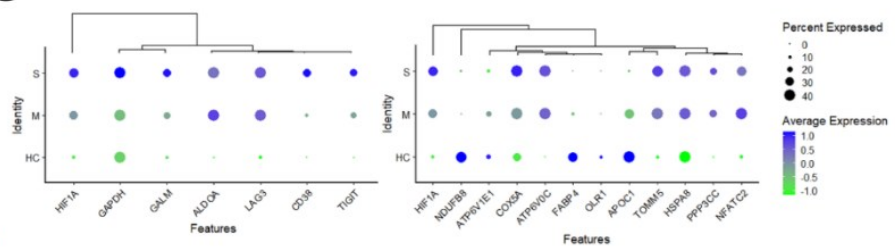

D

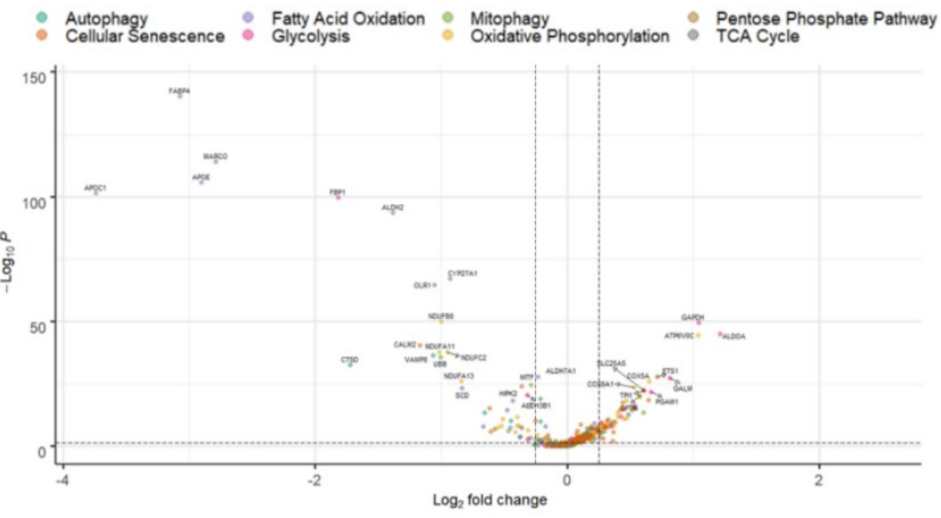

938

939 


\section{CD4 Trajectory}

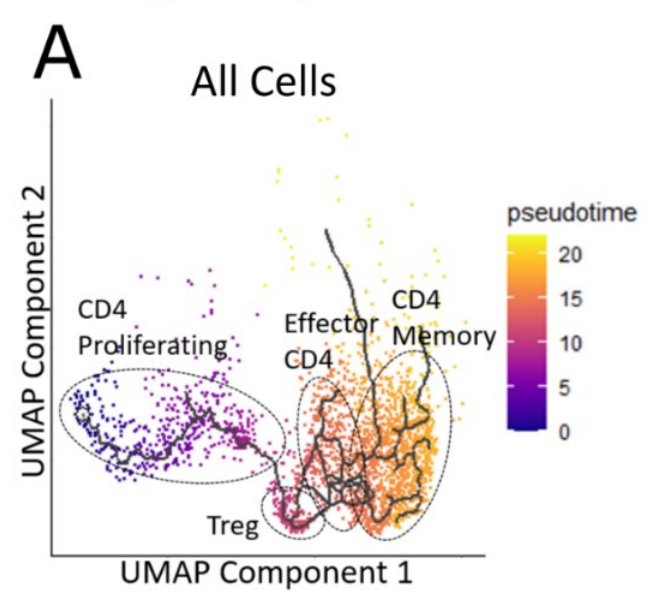

B
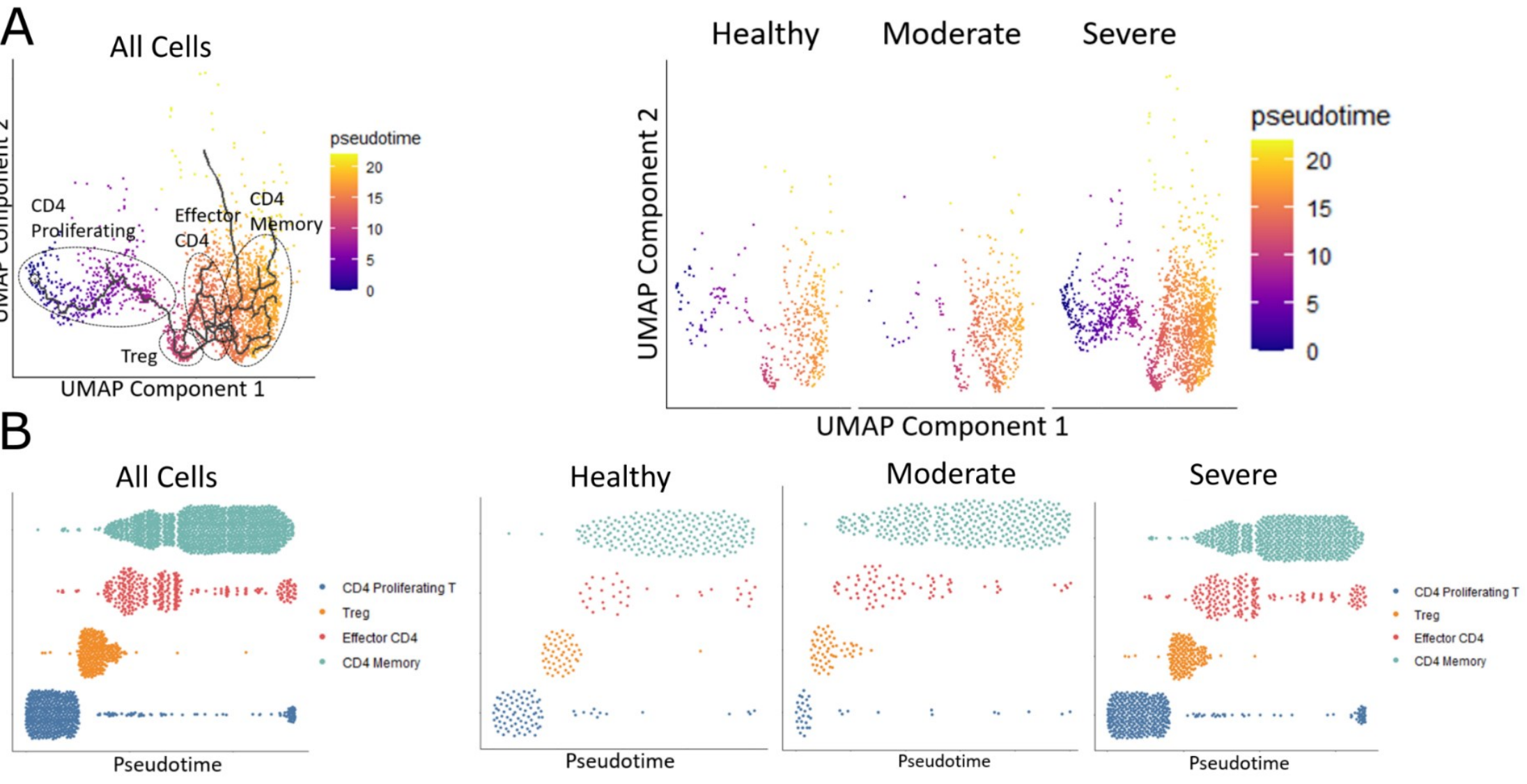

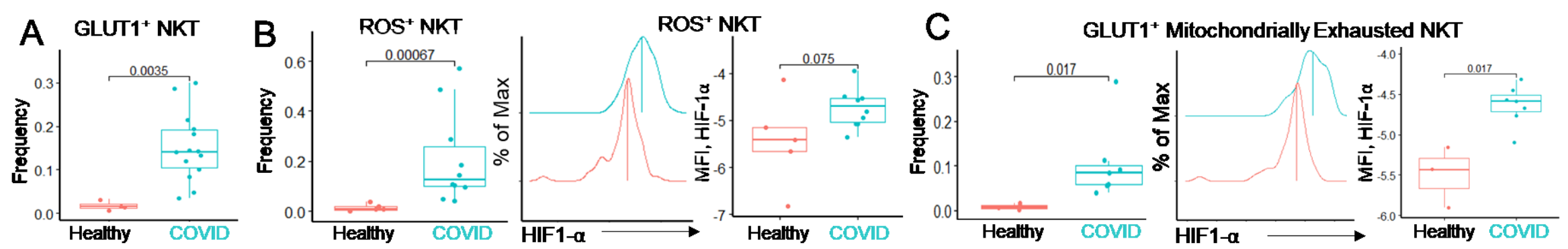

D
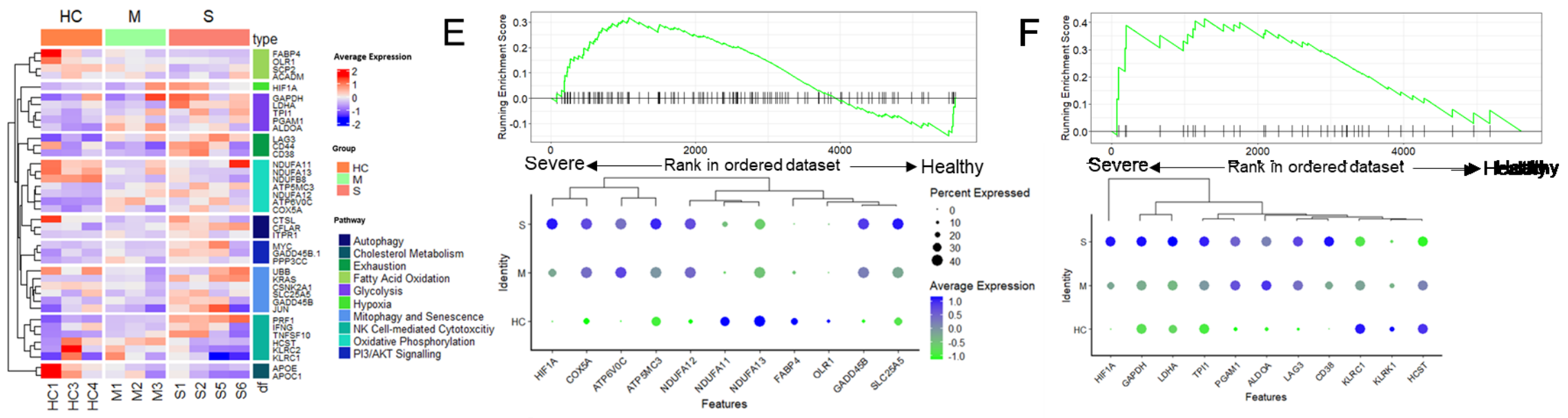


\section{Supplementary Table 1}

\begin{tabular}{ll}
\hline Cell type & Panel for metabolic phenotype-based clustering \\
\hline CTL & APOE, APOC1, FABP4, MARCO, NFUFB8, NDUFC2, NDUFA11, ATP5MC2, \\
& COX5A, ATP6V0C, CD38, LAG3, HIF1A, CFLAR, GABARAP, HSPA8, TOMM5, \\
& OPTN, USP15, NFATC2, PPP3CC, CCND3, GAPDH, GALM, ALDOA \\
\hline CD8 & APOE, APOC, OLR1, MARCO, FABP4, GABARAP, CFLAR, HSPA8, CCND3, \\
Memory & PPP3CC, NFATC2, DGLUCY, GLUD1, CD38, TIGIT, LAG3, GAPDH, GALM, \\
& GPI, ALDOA, HIF1A, TOMM5, USP15, OPTN, NDUFB8, OSP15, OPTN, \\
& NDUFB8, ATP6V1E1, NDUFA1, ATP5MC2, COX5A, ATP6VOC \\
\hline Epithelial & IGFBP3, CDKN1A, GADD45B, ADH1C, ALDOA, GAPDH, PCK2, ENO1, \\
& ALDH1A3, GABARAP, SQSTM1, HIF1A, CTSB, CTSL, NDUFC2, NDUFB8, \\
& ATP5PO, UBB, MDH1, SDHC, ACADM, ACI2, SLC27A2, FABP6, APOC1, \\
& VDAC3 \\
\hline NKT & FABP4, OLR1, SCP2, ACADM, HIF1A, GAPDH, LDHA, TPI1, PGAM1, ALDOA, \\
& LAG3, CD44, CD38, NDUFA11, NDUFA13, NDUFB8, ATp5MC2, NDUFA12, \\
& ATP6V0C, COX5A, CTSL, CFLAR, ITPR1, UBB, KRAS, CSNK2A1, SLC25A5, \\
& GAFF45B, JUN, PRF1, IFNG, TNFSF10, HCST, LRC2, KLRC1, APOE, APOC1 \\
\hline
\end{tabular}

\section{Supplementary Table 2}

\begin{tabular}{|c|c|c|c|c|c|}
\hline & $\begin{array}{l}\text { Glycolysis } \\
\text { Module Score }\end{array}$ & $\begin{array}{l}\text { FAO Module } \\
\text { Score }\end{array}$ & $\begin{array}{l}\text { HLA Class } 2 \\
\text { Signaling } \\
\text { Module Score }\end{array}$ & $\begin{array}{l}\text { Type } 1 \\
\text { Interferon } \\
\text { Module Score }\end{array}$ & $\begin{array}{l}\text { NfKB } \\
\text { Module } \\
\text { Score }\end{array}$ \\
\hline $\begin{array}{l}\text { CD8 } \\
\text { Memory }\end{array}$ & $\begin{array}{l}\text { GAPDH, } \\
\text { GALM, GPI, } \\
\text { ALDOA }\end{array}$ & $\begin{array}{l}\text { OLR, } \\
\text { MARCO, } \\
\text { FABP4 }\end{array}$ & & & \\
\hline Epithelial & $\begin{array}{l}\text { ADH1C, } \\
\text { ALDOA, } \\
\text { GAPDH, } \\
\text { PCK2, ENO1, } \\
\text { ALDH1A3 }\end{array}$ & $\begin{array}{l}\text { ACADM, } \\
\text { ECI2, } \\
\text { SLC27A2, } \\
\text { FABP6 }\end{array}$ & $\begin{array}{l}\text { HLA-DRA, } \\
\text { HLA-DPA1, } \\
\text { HLA-DMA, } \\
\text { DYNLL1 }\end{array}$ & $\begin{array}{l}\text { IFITM1, } \\
\text { IFITM2, IFIT1, } \\
\text { MX1, IFITM2 }\end{array}$ & $\begin{array}{l}\text { SQSTM1, } \\
\text { GADD45B, } \\
\text { NFKBIA, } \\
\text { RELB }\end{array}$ \\
\hline
\end{tabular}

\section{Supplementary Table 3}

\section{PBMC Cell Population}

\section{Surface Markers Used for Identification}

\begin{tabular}{ll}
\hline Cytotoxic T Lymphocytes & CD8, GZMB \\
\hline CD8 Central Memory Cells & CD8, CD62L, and CCR7 \\
\hline CD8 Transitional Memory Cells & CD8, CD62L \\
\hline CD8 Effector Memory Cells & CD8, CCR7, GZMB \\
\hline NK Cells & CD56 \\
\hline NKT Cells & CD56, CD8 \\
\hline CD62L ${ }^{+}$NK Cells & CD56, CD62L \\
\hline
\end{tabular}




\begin{tabular}{lll}
\hline Reagent/Resource & Source & Identifier \\
\hline Anti-human LAG-3 PE/Dazzle & BioLegend & $\begin{array}{l}\text { Clone- } \\
11 C 3 C 65 / 369331\end{array}$ \\
\hline Anti-human CD62L PerCP/Cy 5.5 & BioLegend & $\begin{array}{l}\text { Clone DREG- } \\
56 / 304823\end{array}$ \\
\hline Anti-human CCR7 BV510 & BioLegend & Clone \\
& & G043H7/353231 \\
\hline Anti-human/mouse Granzyme B PE/Cy7 & BioLegend & Clone \\
& & QA16A02/372213 \\
\hline Anti-human CD56 BV650 & BioLegend & Clone \\
& & HCD56/318343 \\
\hline Anti-human GLUT-1 AF700 & R\&D Systems & Clone 202915/ \\
& & FAB1418N \\
\hline Anti-VDAC & Alomone Labs & avc-001-50 mcl \\
\hline CD8 APC/Cy7 & & \\
\hline Anti-human/mouse HIF-1 APC & R\&D Systems & Clone 248182/ \\
& & IC1935A \\
\hline H2DCFDA & ThermoFisher & D399 \\
\hline
\end{tabular}

951

952 
1001

1002

1003

1004

1005

1006

1007

1008

1009

1010

1011

1012

1013

1014

1015

\section{References}

1 Zhu, N. et al. A Novel Coronavirus from Patients with Pneumonia in China, 2019. N Engl J Med 382, 727-733, doi:10.1056/NEJMoa2001017 (2020).

2 Team, I. C.-F. Modeling COVID-19 scenarios for the United States. Nat Med, doi:10.1038/s41591-020-1132-9 (2020).

3 Madhi, S. A. et al. Efficacy of the ChAdOx1 nCoV-19 Covid-19 Vaccine against the B.1.351 Variant. N Engl J Med, doi:10.1056/NEJMoa2102214 (2021).

4 Song, J. W. et al. Omics-Driven Systems Interrogation of Metabolic Dysregulation in COVID-19 Pathogenesis. Cell Metab, doi:10.1016/j.cmet.2020.06.016 (2020).

5 Codo, A. C. et al. Elevated Glucose Levels Favor SARS-CoV-2 Infection and Monocyte Response through a HIF-1alpha/Glycolysis-Dependent Axis. Cell Metab, doi:10.1016/j.cmet.2020.07.007 (2020).

6 Xie, J. et al. Metabolic Syndrome and COVID-19 Mortality Among Adult Black Patients in New Orleans. Diabetes Care, doi:10.2337/dc20-1714 (2020).

7 Wu, D. et al. Plasma metabolomic and lipidomic alterations associated with COVID-19. Nat/ Sci Rev 7, 1157-1168, doi:10.1093/nsr/nwaa086 (2020).

8 Siska, P. J. et al. Metabolic stress and disease-stage specific basigin expression of peripheral blood immune cell subsets in COVID-19 patients. medRxiv, 2020.2009.2018.20194175, doi:10.1101/2020.09.18.20194175 (2020).

9 Lim, A. R., Rathmell, W. K. \& Rathmell, J. C. The tumor microenvironment as a metabolic barrier to effector T cells and immunotherapy. Elife 9, doi:10.7554/eLife.55185 (2020).

10 Kuba, K. et al. A crucial role of angiotensin converting enzyme 2 (ACE2) in SARS coronavirusinduced lung injury. Nat Med 11, 875-879, doi:10.1038/nm1267 (2005).

$11 \mathrm{Li}, \mathrm{X}$. C. et al. Risk factors for severity and mortality in adult COVID-19 inpatients in Wuhan. J Allergy Clin Immun 146, 110-118, doi:10.1016/j.jaci.2020.04.006 (2020).

12 Han, Y. et al. Lactate dehydrogenase, an independent risk factor of severe COVID-19 patients: a retrospective and observational study. Aging (Albany NY) 12, 11245-11258, doi:10.18632/aging.103372 (2020).

13 Huang, C. et al. Clinical features of patients infected with 2019 novel coronavirus in Wuhan, China. Lancet 395, 497-506, doi:10.1016/S0140-6736(20)30183-5 (2020).

14 Zhang, W. et al. Lactate Is a Natural Suppressor of RLR Signaling by Targeting MAVS. Cell 178, 176-189 e115, doi:10.1016/j.cell.2019.05.003 (2019).

15 Haas, R. et al. Lactate Regulates Metabolic and Pro-inflammatory Circuits in Control of T Cell Migration and Effector Functions. PLoS Biol 13, e1002202, doi:10.1371/journal.pbio.1002202 (2015).

16 Maucourant, C. et al. Natural killer cell immunotypes related to COVID-19 disease severity. Sci Immunol 5, doi:10.1126/sciimmunol.abd6832 (2020).

17 Westmeier, J. et al. Impaired Cytotoxic CD8(+) T Cell Response in Elderly COVID-19 Patients. mBio 11, doi:10.1128/mBio.02243-20 (2020).

18 Zhang, J. Y. et al. Single-cell landscape of immunological responses in patients with COVID-19. Nat Immunol 21, 1107-1118, doi:10.1038/s41590-020-0762-x (2020).

19 Macintyre, A. N. et al. The glucose transporter Glut1 is selectively essential for CD4 T cell activation and effector function. Cell Metab 20, 61-72, doi:10.1016/j.cmet.2014.05.004 (2014).

20 Sukumar, M. et al. Inhibiting glycolytic metabolism enhances CD8+ T cell memory and antitumor function. J Clin Invest 123, 4479-4488, doi:10.1172/JCI69589 (2013).

21 Duette, G. et al. Induction of HIF-1alpha by HIV-1 Infection in CD4(+) T Cells Promotes Viral Replication and Drives Extracellular Vesicle-Mediated Inflammation. mBio 9, doi:10.1128/mBio.00757-18 (2018).

$22 \mathrm{Kim}$, J. et al. VDAC oligomers form mitochondrial pores to release mtDNA fragments and promote lupus-like disease. Science 366, 1531-1536, doi:10.1126/science.aav4011 (2019).

23 Waickman, A. T. \& Powell, J. D. mTOR, metabolism, and the regulation of T-cell differentiation and function. Immunol Rev 249, 43-58, doi:10.1111/j.1600-065X.2012.01152.x (2012).

24 Tullius, S. G. et al. NAD+ protects against EAE by regulating CD4+ T-cell differentiation. Nat Commun 5, 5101, doi:10.1038/ncomms6101 (2014). 
1016

1017

1018

1019

1020

1021

1022

1023

1024

1025

1026

1027

1028

1029

1030

1031

1032

1033

1034

1035

1036

1037

1038

1039

1040

1041

1042

1043

1044

1045

1046

1047

1048

1049

1050

1051

1052

1053

1054

1055

1056

1057

1058

1059

1060

1061

1062

1063

1064

1065

1066

1067

1068

1069

25 Chatterjee, S. et al. CD38-NAD(+)Axis Regulates Immunotherapeutic Anti-Tumor T Cell Response. Cell Metab 27, 85-100 e108, doi:10.1016/j.cmet.2017.10.006 (2018).

26 Angin, M. et al. Metabolic plasticity of HIV-specific CD8(+) T cells is associated with enhanced antiviral potential and natural control of HIV-1 infection. Nat Metab 1, 704-716, doi:10.1038/s42255-019-0081-4 (2019).

27 Schenkel, J. M. \& Masopust, D. Tissue-resident memory T cells. Immunity 41, 886-897, doi:10.1016/j.immuni.2014.12.007 (2014).

28 van der Windt, G. J. \& Pearce, E. L. Metabolic switching and fuel choice during T-cell differentiation and memory development. Immunol Rev 249, 27-42, doi:10.1111/j.1600065X.2012.01150.x (2012).

29 Ayres, J. S. A metabolic handbook for the COVID-19 pandemic. Nat Metab 2, 572-585, doi:10.1038/s42255-020-0237-2 (2020).

30 Paget, C. \& Trottein, F. Role of type 1 natural killer T cells in pulmonary immunity. Mucosal Immunol 6, 1054-1067, doi:10.1038/mi.2013.59 (2013).

31 Everts, B. et al. TLR-driven early glycolytic reprogramming via the kinases TBK1-IKKvarepsilon supports the anabolic demands of dendritic cell activation. Nat Immunol 15, 323-332, doi:10.1038/ni.2833 (2014).

32 Juelke, K. et al. CD62L expression identifies a unique subset of polyfunctional CD56dim NK cells. Blood 116, 1299-1307, doi:10.1182/blood-2009-11-253286 (2010).

33 Zhang, X. et al. Viral and host factors related to the clinical outcome of COVID-19. Nature, doi:10.1038/s41586-020-2355-0 (2020).

34 Certo, M., Tsai, C. H., Pucino, V., Ho, P. C. \& Mauro, C. Lactate modulation of immune responses in inflammatory versus tumour microenvironments. Nat Rev Immunol, doi:10.1038/s41577-020-0406-2 (2020).

35 McLane, L. M., Abdel-Hakeem, M. S. \& Wherry, E. J. CD8 T Cell Exhaustion During Chronic Viral Infection and Cancer. Annu Rev Immunol 37, 457-495, doi:10.1146/annurev-immunol-041015055318 (2019).

36 Cascone, T. et al. Increased Tumor Glycolysis Characterizes Immune Resistance to Adoptive T Cell Therapy. Cell Metab 27, 977-987 e974, doi:10.1016/j.cmet.2018.02.024 (2018).

37 Hariyanto, T. I. \& Kurniawan, A. Metformin use is associated with reduced mortality rate from coronavirus disease 2019 (COVID-19) infection. Obes Med 19, 100290, doi:10.1016/j.obmed.2020.100290 (2020).

38 Drosten, C. et al. Identification of a novel coronavirus in patients with severe acute respiratory syndrome. N Engl J Med 348, 1967-1976, doi:10.1056/NEJMoa030747 (2003).

39 Singh, Y. et al. SARS-CoV-2 infection paralyzes cytotoxic and metabolic functions of immune cells. bioRxiv, 2020.2009.2004.282780, doi:10.1101/2020.09.04.282780 (2020).

40 De Biasi, S. et al. Marked T cell activation, senescence, exhaustion and skewing towards TH17 in patients with COVID-19 pneumonia. Nat Commun 11, 3434, doi:10.1038/s41467-020-17292-4 (2020).

41 Thompson, E. A. et al. Metabolic programs define dysfunctional immune responses in severe COVID-19 patients. medRxiv, 2020.2009.2010.20186064, doi:10.1101/2020.09.10.20186064 (2020).

42 Kang, C. K. et al. Aberrant hyperactivation of cytotoxic T-cell as a potential determinant of COVID-19 severity. Int J Infect Dis 97, 313-321, doi:10.1016/j.ijid.2020.05.106 (2020).

43 Jouan, Y. et al. Phenotypical and functional alteration of unconventional T cells in severe COVID19 patients. J Exp Med 217, doi:10.1084/jem.20200872 (2020).

44 Cerwenka, A. \& Lanier, L. L. Natural killer cell memory in infection, inflammation and cancer. Nat Rev Immunol 16, 112-123, doi:10.1038/nri.2015.9 (2016).

45 Group, R. C. et al. Dexamethasone in Hospitalized Patients with Covid-19 - Preliminary Report. N Engl J Med, doi:10.1056/NEJMoa2021436 (2020).

$46 \mathrm{Ma}, \mathrm{R}$. et al. Switch of glycolysis to gluconeogenesis by dexamethasone for treatment of hepatocarcinoma. Nat Commun 4, 2508, doi:10.1038/ncomms3508 (2013).

47 Cron, R. Q. COVID-19 cytokine storm: targeting the appropriate cytokine. Lancet Rheumatol 3, e236-e237, doi:10.1016/S2665-9913(21)00011-4 (2021). 
1070

1071

1072

1073

1074

1075

1076

1077

1078

1079

1080

1081

1082

1083

1084

1085

1086

1087

1088

1089

1090

1091

1092

1093

1094

1095

1096

1097

1098

1099

1100

1101

1102

1103

1104

1105

1106

1107

1108

1109

1110

1111

1112

1113

1114

1115

1116

1117

1118

1119

1120

1121

1122

48 Bhatt, A. N. et al. Glycolytic inhibitor 2-Deoxy-D-glucose attenuates SARS-CoV-2 multiplication in host cells and weakens the infective potential of progeny virions. bioRxiv, 2021.2006.2012.448175, doi:10.1101/2021.06.12.448175 (2021).

49 Wu, D. et al. Type 1 Interferons Induce Changes in Core Metabolism that Are Critical for Immune Function. Immunity 44, 1325-1336, doi:10.1016/j.immuni.2016.06.006 (2016).

50 Park, A. \& Iwasaki, A. Type I and Type III Interferons - Induction, Signaling, Evasion, and Application to Combat COVID-19. Cell Host Microbe 27, 870-878, doi:10.1016/j.chom.2020.05.008 (2020).

51 Yu, Y. R. et al. Disturbed mitochondrial dynamics in CD8(+) TILs reinforce T cell exhaustion. Nat Immunol 21, 1540-1551, doi:10.1038/s41590-020-0793-3 (2020).

52 Xie, J. et al. Association Between Hypoxemia and Mortality in Patients With COVID-19. Mayo Clin Proc 95, 1138-1147, doi:10.1016/j.mayocp.2020.04.006 (2020).

53 Tillett, R. L. et al. Genomic evidence for reinfection with SARS-CoV-2: a case study. Lancet Infect Dis 21, 52-58, doi:10.1016/S1473-3099(20)30764-7 (2021).

54 Egawa, T. \& Bhattacharya, D. Regulation of metabolic supply and demand during B cell activation and subsequent differentiation. Curr Opin Immunol 57, 8-14, doi:10.1016/j.coi.2018.10.003 (2019).

55 Kaneko, N. et al. Loss of Bcl-6-Expressing T Follicular Helper Cells and Germinal Centers in COVID-19. Cell 183, 143-157 e113, doi:10.1016/j.cell.2020.08.025 (2020).

56 Woodruff, M. C. et al. Extrafollicular B cell responses correlate with neutralizing antibodies and morbidity in COVID-19. Nat Immunol 21, 1506-1516, doi:10.1038/s41590-020-00814-z (2020).

57 Dan, J. M. et al. Immunological memory to SARS-CoV-2 assessed for up to 8 months after infection. Science 371, doi:10.1126/science.abf4063 (2021).

58 Gaebler, C. et al. Evolution of Antibody Immunity to SARS-CoV-2. bioRxiv, 2020.2011.2003.367391, doi:10.1101/2020.11.03.367391 (2020).

59 Lucas, C. et al. Longitudinal analyses reveal immunological misfiring in severe COVID-19. Nature, doi:10.1038/s41586-020-2588-y (2020).

60 Nightingale Health, U. K. B. I., Julkunen, H., Cichonska, A., Slagboom, P. E. \& Wurtz, P. Metabolic biomarker profiling for identification of susceptibility to severe pneumonia and COVID19 in the general population. Elife 10, doi:10.7554/eLife.63033 (2021).

61 Hahne, F. et al. flowCore: a Bioconductor package for high throughput flow cytometry. BMC Bioinformatics 10, 106, doi:10.1186/1471-2105-10-106 (2009).

62 Finak, G. et al. OpenCyto: an open source infrastructure for scalable, robust, reproducible, and automated, end-to-end flow cytometry data analysis. PLoS Comput Biol 10, e1003806, doi:10.1371/journal.pcbi.1003806 (2014).

63 Chevrier, S. et al. Compensation of Signal Spillover in Suspension and Imaging Mass Cytometry. Cell Syst 6, 612-620 e615, doi:10.1016/j.cels.2018.02.010 (2018).

64 Van Gassen, S., Gaudilliere, B., Angst, M. S., Saeys, Y. \& Aghaeepour, N. CytoNorm: A Normalization Algorithm for Cytometry Data. Cytometry A 97, 268-278, doi:10.1002/cyto.a.23904 (2020).

65 Van Gassen, S. et al. FlowSOM: Using self-organizing maps for visualization and interpretation of cytometry data. Cytometry A 87, 636-645, doi:10.1002/cyto.a.22625 (2015).

66 Liao, M. et al. Single-cell landscape of bronchoalveolar immune cells in patients with COVID-19. Nat Med 26, 842-844, doi:10.1038/s41591-020-0901-9 (2020).

67 Morse, C. et al. Proliferating SPP1/MERTK-expressing macrophages in idiopathic pulmonary fibrosis. Eur Respir J 54, doi:10.1183/13993003.02441-2018 (2019).

68 Butler, A., Hoffman, P., Smibert, P., Papalexi, E. \& Satija, R. Integrating single-cell transcriptomic data across different conditions, technologies, and species. Nat Biotechnol 36, 411-+, doi:10.1038/nbt.4096 (2018).

69 Stuart, T. et al. Comprehensive Integration of Single-Cell Data. Cell 177, 1888-+, doi:10.1016/j.cell.2019.05.031 (2019).

70 Cao, J. et al. The single-cell transcriptional landscape of mammalian organogenesis. Nature 566, 496-502, doi:10.1038/s41586-019-0969-x (2019). 
112371 Zhou, G. Y. et al. NetworkAnalyst 3.0: a visual analytics platform for comprehensive gene expression profiling and meta-analysis. Nucleic Acids Res 47, W234-W241, doi:10.1093/nar/gkz240 (2019).

1127

1128

1129

1130

1131

1132

72 Yu, G. Using ggtree to Visualize Data on Tree-Like Structures. Curr Protoc Bioinformatics 69, e96, doi:10.1002/cpbi.96 (2020).

73 Mathewson, N. D. et al. Corrigendum: Gut microbiome-derived metabolites modulate intestinal epithelial cell damage and mitigate graft-versus-host disease. Nat Immunol 17, 1235, doi:10.1038/ni1016-1235b (2016).

74 Kuleshov, M. V. et al. Enrichr: a comprehensive gene set enrichment analysis web server 2016 update. Nucleic Acids Res 44, W90-97, doi:10.1093/nar/gkw377 (2016). 